\title{
Zielorientierungen und Leistungsmotivation angehender Lehrkräfte zu Studienbeginn - eine Profilanalyse
}

\author{
Nina Glutsch (D) Johannes König (D) Martin Rothland
}

Eingegangen: 29. April 2019 / Überarbeitet: 27. März 2020 / Angenommen: 16. Juli 2020 / Online publiziert: 6. August 2020

(C) Der/die Autor(en) 2020

Zusammenfassung Im Vergleich zu den vielfach untersuchten Leistungsmotiven und Zielorientierungen von Schülerinnen und Schülern sind diese bei Lehramtsstudierenden bisher eher selten zum Gegenstand empirischer Forschung gemacht worden, obwohl sie für die Kompetenzentwicklung und das Leistungsstreben angehender Lehrpersonen im Studium eine wichtige Rolle spielen. Vor diesem Hintergrund untersucht der vorliegende Beitrag Zielorientierungen und die Leistungsmotive Hoffnung auf Erfolg und Angst vor Misserfolg von 1365 Studierenden der Lehrämter für die Sekundarstufen I und II in ihrem ersten Fachsemester mittels einer latenten Profilanalyse. Die Profile werden anhand weiterer motivationaler Merkmale und dem pädagogischen Wissen der Studierenden validiert sowie auf ihre Zusammensetzung hinsichtlich verschiedener Fächerkombinationen analysiert. Die Ergebnisse legen eine Dreiklassenlösung nahe, wobei sich die Profile nicht nach Fächerdomänen unterscheiden. Die Hälfte der Studierenden lässt sich einem sowohl lern- als auch leistungszielorientierten Profil zuordnen. Am zweithäufigsten ist ein stark lernzielorientiertes Profil vertreten. Die kleinste Profilgruppe umfasst Studierende mit einer ausgeprägt arbeitsvermeidenden Haltung.

Dr. N. Glutsch (凶) · Prof. Dr. J. König

Humanwissenschaftliche Fakultät, Department für Erziehungs- und Sozialwissenschaften,

Empirische Schulforschung, Schwerpunkt Quantitative Methoden, Universität zu Köln,

Gronewaldstr. 2a, 50931 Köln, Deutschland

E-Mail: nina.glutsch@uni-koeln.de

Prof. Dr. J. König

E-Mail: johannes.koenig@uni-koeln.de

Prof. Dr. M. Rothland

Fachbereich 06, Institut für Erziehungswissenschaft, Westfälische Wilhelms-Universität Münster,

Bispinghof 5/6, 48143 Münster, Deutschland

E-Mail: Martin.Rothland@uni-muenster.de 
Schlüsselwörter Zielorientierungen und Leistungsmotivation ·

Lehramtsstudierende $\cdot$ Latente Profilanalyse $\cdot$ Fachdifferenzen

\title{
Pre-service teachers' achievement goals and achievement motivation at the beginning of their studies-a profile analysis
}

\begin{abstract}
Pre-service teachers' achievement goals and achievement motivation have not yet been as intensely examined as those of school students. However, such goals and motivation can play an important role concerning pre-service teachers' professional development and goal aspirations during initial teacher education. Based on the achievement goals and achievement motivations of 1365 pre-service lower and upper secondary teachers in their first semester at universities, latent profiles are identified and validated with additional motivational characteristics and students' general pedagogical knowledge. Furthermore, the profiles are analyzed concerning their distribution of different subject domains. Results suggest a three-class solution, whereby the classes do not show differences concerning the subject domains. $50 \%$ of the students show strong mastery goals as well as performance goals. The second most common profile comprises students who mainly show mastery goals. The smallest profile group comprises students with strong work avoidance goal orientations.
\end{abstract}

Keywords Achievement goals and achievement motivation - Latent profile analysis $\cdot$ Pre-service teachers $\cdot$ Subject domain differences

\section{Einleitung}

Zielorientierungen gelten als motivationale Merkmale, die generell das Leistungsstreben und Verhalten von Personen in Lern- und Leistungssituationen beeinflussen können (Harackiewicz et al. 1997; Spinath et al. 2002). Das Konzept der Zielorientierungen wurde zunächst vor allem bei Schüler*innen untersucht, fand aber in den vergangenen Jahren auch Einzug in die Forschung zur Motivation von Lehrer*innen. Der Lern- und Arbeitskontext Schule enthält für Lehrkräfte vielfältige Situationen, die Lernen, Leistung und einen hohen Grad der Motivation erfordern (Butler 2007; Kunter 2014). Auch angehende Lehrkräfte finden sich in ihrem Studium und während praktischer Phasen u.a. in Form von Prüfungen oder ersten eigenen Lehrerfahrungen vielfältigen Lern- und Leistungssituationen ausgesetzt. Zielorientierungen von angehenden Lehrkräften im Studium wurden bis auf wenige Studien (vgl. Schöne et al. 2004; Malmberg 2008; Tönjes und Dickhäuser 2009; Fasching et al. 2010) gleichwohl selten untersucht. Gerade zu Beginn des Studiums sind Lernund Leistungsmotive jedoch von Bedeutung, da sie als wichtige Prädiktoren für Studienleistungen und Kompetenzentwicklung gelten. Daher werden in der vorliegenden Untersuchung Studierende im ersten Fachsemester aus den Studiengängen der Lehrämter für die Sekundarstufe I und II an 18 verschiedenen Hochschulen in Deutschland fokussiert. Die Ergebnisse können Einblick in die motivationalen Voraussetzungen von Lehramtsstudierenden geben, die für Lern- und Leistungskon- 
texte und somit die Entwicklung der professionellen Kompetenz entscheidend sind (Schiefele und Urhahne 2000).

Zielorientierungen (Lernziel-, Annäherungs-Leistungs- und Vermeidungs-Leistungszielorientierungen) werden nach dem hierarchischen Modell von Elliot und Church (1997, S. 219) als kognitiv-dynamische Manifestationen der tieferliegenden Leistungs- und Misserfolgsmotive in Form von Hoffnung auf Erfolg und Furcht vor Misserfolg beschrieben. Einige Studien haben Zielorientierungen deshalb in Beziehung zu den Leistungsmotiven gesetzt (Elliot und McGregor 2001; Spinath et al. 2002; König und Rothland 2013). So folgt auch der vorliegende Beitrag dieser Modellvorstellung und nimmt beide Konstrukte im Rahmen einer latenten Profilanalyse in den Blick. Belegt ist mittlerweile, dass Personen mehrere, auch unterschiedliche Ziele gleichzeitig verfolgen können (vgl. z. B. Barron und Harackiewicz 2001; Schwinger und Wild 2006). Die Ausprägung und das Zusammenspiel von Zielorientierungen und Leistungsmotiven soll deshalb mittels eines personenzentrierten Ansatzes erfasst werden, sodass Personen hinsichtlich ihrer unterschiedlichen Zielorientierungen und Leistungsmotive klassifiziert und Profile identifiziert werden. Verschiedene Profilanalysen mit dem Fokus auf Zielorientierungen zeigen heterogene Befunde und eine unterschiedliche Anzahl an Profilen (vgl. Wormington und Linnenbrink-Garcia 2017). Allgemeine Leistungsmotivation und Zielorientierungen von Lehramtsstudierenden interagieren und haben jeweils Effekte auf Lernzuwachs, Leistung und weitere affektiv-motivationale Merkmale. Daher gilt es, beide Konstrukte nach dem hierarchischen Modell (Elliot und Church 1997) in einem personenzentrierten Ansatz zu integrieren und bestehende Untersuchungen zu angehenden Lehrkräften (vgl. Fasching et al. 2010; Affolter 2016) oder Studierenden generell (Pastor et al. 2007) durch die vorliegende Studie zu erweitern.

Untersucht wird in dem vorliegenden Beitrag zum einen die Frage, welche Profile sich unter Einbeziehung der Lern- und Leistungszielorientierungen sowie Leistungs- und Misserfolgsmotive von Lehramtsstudierenden zeigen. Zum anderen wird der Frage nachgegangen, inwiefern sich die Profile validieren lassen. Dabei stehen Effekte auf weitere motivationale Merkmale sowie das pädagogische Wissen der Studierenden im Mittelpunkt und somit Konstrukte, die mit Lern- und Leistungsmotiven und Zielorientierungen in Verbindung stehen oder ihre Folge bzw. ihre Konsequenz darstellen. Die Analyse bezieht Studierende der Lehrämter für Sekundarstufe I und II ein, die jeweils zwei Fächer studieren. Dies ermöglicht, zusätzlich die Verteilung der Fächerdomänen zu berücksichtigen und der Frage nachzugehen, ob sich Zielorientierungen und Leistungsmotive eher kontextunabhängig oder eher domänenspezifisch abbilden (Köller 1998; Malmberg 2008; Watermann 2013).

\section{Theoretischer Hintergrund}

\subsection{Lern-/Leistungszielorientierungen und Allgemeine Leistungsmotivation im Kontext der Lehrer*innenbildung}

Sowohl das Lehramtsstudium als auch der spätere Lehrberuf gelten als leistungsthematische Umgebungen (Dickhäuser et al. 2007). Schule wird von Butler (2007, 
S. 242) auch als ,achievement arena“ bezeichnet, in der Lehrkräfte ihren Beruf erfolgreich ausüben möchten, sich aber in der Art und Weise unterscheiden können, wie sie dies tun und welche Ziele sie dabei sowohl für ihre Schüler*innen als auch für sich selbst verfolgen. Bereits Lehramtsstudierende unterschieden sich in ihren Zielen und motivationalen Merkmalen bereits zu Studienbeginn voneinander. Im öffentlichen Diskurs werden ihre motivationalen und kognitiven Eingangsmerkmale häufig thematisiert. Vor allem in Bezug auf die ,richtigen“ Eingangsvoraussetzungen stellt sich die Frage, welche Studierenden zu solchen Lehrkräften werden, die auf die Lernleistung ihrer Schüler*innen positiven Einfluss nehmen (Rothland und Terhart 2011, S. 636). Zu den Eingangsvoraussetzungen zählen auch motivationale Merkmale, die in einer Wirkungskette neben kognitiven Merkmalen untersucht werden (Kunter et al. 2011). Für die Entwicklung der professionellen Kompetenz von (angehenden) Lehrkräften ist eine adaptive Lern- und Leistungsmotivation ausschlaggebend, um Lernanlässe und Lernangebote gewinnbringend zu nutzen. In der vorliegenden Studie stehen daher im Zusammenhang mit der professionellen Kompetenzentwicklung auf Seiten der motivationalen Orientierungen zum einen die Zielorientierungen und zum anderen die Leistungsmotivation von Lehramtsstudierenden im Mittelpunkt.

Generell können Zielorientierungen als ,motivationale Ausrichtung von Personen in Lern- und Leistungskontexten“ (Schöne et al. 2004, S. 94) definiert werden. Das Konzept der Zielorientierungen, wie es in der vorliegenden Studie untersucht wird, umfasst die Bereiche Lernziele und Leistungsziele (mastery goals und performance goals nach Dweck 1986), die wiederum nach dem hierarchischen Modell von Elliot und Harackiewicz (1996; vgl. auch Elliot und Church 1997) in AnnäherungsLeistungsziele und Vermeidungs-Leistungsziele differenziert werden. Dabei streben lernzielorientierte Personen nach Kompetenzerweiterung, also nach Lernzuwachs und der Entwicklung und Verbesserung eigener Fähigkeiten. Leistungszielorientierte Personen dagegen wollen die eigene Kompetenz nach außen demonstrieren (Annäherungs-Leistungszielorientierung) oder Fähigkeitsdefizite verbergen (VermeidungsLeistungszielorientierung) (vgl. Spinath und Schöne 2003; Dickhäuser et al. 2007). Eine weitere Komponente der Zielorientierungen, die wir einbeziehen, ist die Arbeitsvermeidung nach Nicholls (1984), bei der Anstrengung in einer Lern- oder Leistungssituation vermieden werden soll und gleichzeitig ein gesetztes Ziel angestrebt wird.

Befunde aus der Motivationsforschung bei Schüler*innen zeigen, dass vor allem Lernziele positive Prädiktoren für intrinsische Motivation, bessere Leistungen (vgl. z. B. Köller 1998; Robbins et al. 2004) und größeres Interesse am Lerngegenstand (Harackiewicz et al. 1997) sind. Zudem führen lernzielorientierte Personen ihre Leistung eher auf die eigene Anstrengung als auf Fähigkeiten oder Begabungen zurück (Ames 1984). Für Annäherungs-Leistungszielorientierungen liegen dagegen widersprüchliche Ergebnisse vor (Senko und Dawson 2017, S. 574). Sie können nicht nur positive, sondern auch negative Aspekte wie oberflächliche Lernstrategien oder Unsicherheit vorhersagen. So haben Senko und Dawson (2017) in einer Metaanalyse zwei unterschiedlich operationalisierte Ausrichtungen der AnnäherungsLeistungszielorientierungen identifiziert. Ist das Ziel, eigene Kompetenz nach außen zu demonstrieren (,,appearance goals“), wirken sich Annäherungs-Leistungszielori- 


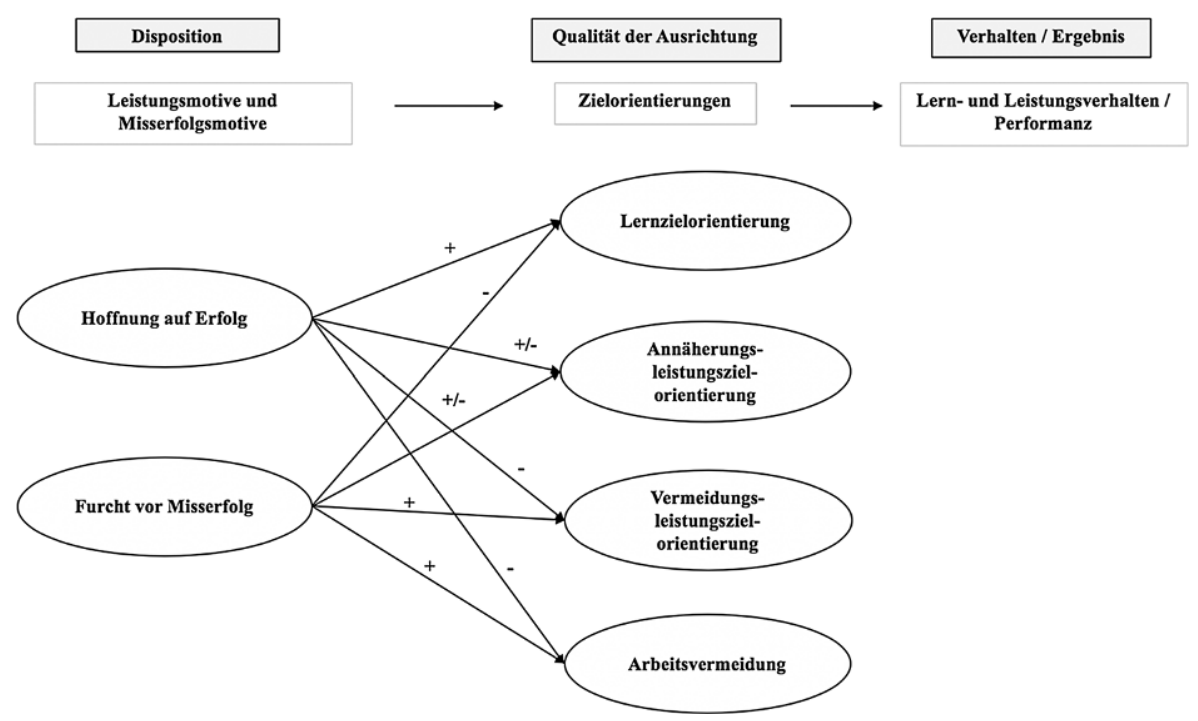

Abb. 1 Hierarchisches Modell der Leistungsmotivation und Zielorientierungen nach Elliot und Church (1997)

entierungen eher maladaptiv etwa auf Lernstrategien aus. Steht aber der Wettbewerb mit Gleichaltrigen im Vordergrund (,normative goals“"), werden beispielsweise effektive Lernstrategien und Selbstvertrauen gestärkt (Senko und Dawson 2017). Annäherungs-Leistungsziele können somit nicht per se als adaptiv oder maladaptiv betrachtet werden. Ihre Wirkung ist vielmehr in Abhängigkeit von der Operationalisierung zu beurteilen. So konnten für Annäherungs-Leistungsziele auch positive Effekte auf gute Leistungen nachgewiesen werden (z. B. $\beta=0,21$, Harackiewicz et al. 1997). Vermeidungs-Leistungsziele gehen indes mit schlechteren Leistungen einher ( $r=-0,23$ für Schüler*innen des Gymnasiums), genau wie hohe Arbeitsvermeidungstendenzen ( $r=0,20$ für Schüler*innen des Gymnasiums, Spinath et al. 2002, S. 29). Schlechtere Leistungen bei Schüler*innen mit einer arbeitsvermeidenden Haltung $(\beta=-0,15)$ wiesen auch Harackiewicz et al. (1997) nach. Umgekehrt konnten Seifert und O'Keefe (2001, S. 86) zeigen, dass einerseits die wahrgenommene Relevanz der Lerninhalte bzw. der Sinn einer Aufgabe die Wahrscheinlichkeit bedeutsam verringert, dass Schüler*innen arbeitsvermeidende Ziele verfolgen $(\beta=-0,76)$, und andererseits die eigene als hoch wahrgenommene Kompetenz Lernzielorientierungen verstärkt $(\beta=0,32)$. Neben Schüler*innen wurden auch Psychologiestudierende untersucht. Hier stellten Elliot und McGregor (1999, S. 638) einen positiven Zusammenhang der Annäherungs-Leistungsziele mit der Leistung im Studium und einen negativen für Vermeidungs-Leistungsziele fest.

Elliot und Church (1997, S. 219) beschreiben Zielorientierungen als „,cognitivedynamic manifestations" der Leistungsmotivation in einem hierarchischen Modell (vgl. Abb. 1). Ob als implizite oder bewusste Bedürfnisse, die Leistungsmotivation bestimmt das Leistungsverhalten einer Person in positiver oder negativer Weise. Sie stellt ein tieferliegendes Merkmal dar und ist den Zielorientierungen vorgeordnet 
(Elliot und Church 1997). Wir untersuchen deshalb zusätzlich zu den Zielorientierungen die Leistungsmotivation als dispositionales Merkmal, das sich aus der Hoffnung auf Erfolg (Leistungsmotiv, Stolz bei Erfolg) und der Furcht vor Misserfolg (Misserfolgsmotiv, Scham bei Versagen) zusammensetzt (Atkinson 1964). Nach Nicholls (1984) trauen sich leistungs- bzw. erfolgsmotivierte Personen mehr zu und sind deshalb anders und stärker motiviert, eine schwierige Aufgabe anzugehen als misserfolgsmotivierte Personen. Diese Beschreibung verdeutlicht noch einmal, dass ein positiver Zusammenhang zwischen dem Erfolgsmotiv und der Lernzielorientierung sowie Annäherungsleistungszielorientierung naheliegt. Elliot und Church (1997) konnten zunächst zeigen, dass bei College-Studierenden des Fachs Psychologie die Hoffnung auf Erfolg unmittelbar mit der Lernzielorientierung $(\beta=0,22)$ und die Furcht vor Misserfolg mit Vermeidungs-Leistungszielen zusammenhängt $(\beta=0,45)$. Für die Annäherungs-Leistungsziele fiel das Ergebnis weniger eindeutig aus, da diese sowohl in der Hoffnung auf Erfolg $(\beta=0,41)$ als auch der Furcht vor Misserfolg $(\beta=0,45)$ begründet lagen.

\subsection{Befunde zu Zielorientierungen angehender Lehrkräfte}

In der Literatur finden sich einige wenige Studien, die sich speziell nur mit Lehramtsstudierenden auseinandersetzen. Bereits belegt werden konnten signifikant positive Zusammenhänge zwischen einer ausgeprägten Lernzielorientierung und der intrinsischen Berufswahlmotivation ( $r=0,48$, Malmberg 2008; $r=0,26$, König und Rothland 2013) sowie fachspezifischen und pädagogischen Berufswahlmotiven (Malmberg 2008; Paulick et al. 2013). Eine höhere Lernzielorientierung ist zudem positiv mit dem pädagogischen Wissen korreliert $(r=0,18)$, eine ausgeprägte Arbeitsvermeidungstendenz negativ ( $r=-0,15$, König und Rothland 2013). Der Zusammenhang von Zielorientierungen und Leistung zeigte sich auch bei angehenden Lehrkräften im Referendariat. Insbesondere eine ausgeprägte Lernzielorientierung kann hier als günstige Voraussetzung für die Entwicklung beruflicher Kompetenzen in dieser Ausbildungsphase gelten ( $r=-0,22$ für den Zusammenhang zwischen Lernzielen und Stresserleben, Fasching et al. 2010, S. 21; $\beta=0,3$ für den Effekt der Lernzielorientierung auf berufliches Engagement, Affolter 2016). Hohe VermeidungsLeistungszielorientierungen dagegen hängen negativ mit den Selbstwirksamkeitserwartungen von Lehrkräften bei Berufseintritt zusammen ( $\beta=-0,32$, Affolter 2016). Denselben Befund konnten Nitsche et al. (2011) für Referendar*innen nachweisen. Ihre Ergebnisse belegen außerdem positive Effekte der Lernzielorientierung und Annäherungs-Leistungszielorientierung auf die Selbstwirksamkeitserwartung. Tönjes et al. (2008) belegten für Studierende, dass hohe Annäherungs-Leistungsziele die eigene Wahrnehmung von Leistungsmangel verringern können $(\beta=-0,45)$ und Vermeidungs-Leistungsziele den wahrgenommenen Leistungsmangel erhöhen $(\beta=0,36)$. Ähnliche Werte fanden fanden die Autor*innen für die Zusammenhänge bei Lehrkräften.

Mit Blick auf Lehrkräfte im Beruf konnten weitere Untersuchungen positive Zusammenhänge zwischen Vermeidungs-Leistungszielen und emotionaler Erschöpfung nachweisen ( $r=0,42$, Tönjes und Dickhäuser 2009, S. 84). Eine ausgeprägte Lernzielorientierung dagegen korreliert mit einem geringeren Burnout-Risiko $(r=-0,22)$ 
und größerem Interesse am Lehren ( $r=0,36$, Retelsdorf et al. 2010). Sie erleichtert auch das Wahrnehmen von Hilfe und das Einholen von Feedback ( $r=0,37$, Butler 2007; vgl. auch Dickhäuser et al. 2007), was wiederum als lernförderlich gilt. Zudem werden ebenfalls Zusammenhänge zwischen den Zielorientierungen der Lehrkräfte, ihrem Unterrichtshandeln und den Effekten auf die Zielorientierungen ihrer Schüler*innen untersucht. Demnach führen hohe Lernzielorientierungen der Lehrkräfte zu ebenfalls positiven Zielorientierungen ihrer Schüler*innen $(\beta=0,20$ für den Effekt der Lehrekräfte-Lernziele auf die Schüler*innen-Annäherungs-Leistungsziele, vgl. Dresel et al. 2013).

Zusammenfassend lässt sich festhalten, dass vor allem für die Lernzielorientierungen positive Zusammenhänge mit sowohl motivational-affektiven als auch kognitiven Merkmalen bestehen. Doch auch für Annäherungs-Leistungsziele haben sich immer wieder positive Effekte nachweisen lassen. Umgekehrt gehen mit höheren Vermeidungs-Leistungszielen auch negative Aspekte wie emotionale Erschöpfung einher. Für die Bildung der Profile in der vorliegenden Studie weisen die hier dargestellten Ergebnisse darauf hin, dass sich verschiedene Profilgruppen finden lassen, die jeweils eindeutige Tendenzen in den unterschiedlichen Zielorientierungen aufweisen.

\subsection{Befunde zur Allgemeinen Leistungsmotivation angehender Lehrkräfte}

Bezugnehmend auf das hierarchische Modell von Elliot und Church (1997) werden im Folgenden einige Befunde zu den Zusammenhängen zwischen den Leistungsmotiven Hoffnung auf Erfolg und Furcht vor Misserfolg und den Zielorientierungen berichtet. Für Lehramts-studierende liegen Befunde vor, die zeigen, dass intrinsische Motive wie das Interesse am Beruf und Unterrichten positive Zusammenhänge mit der Hoffnung auf Erfolg aufweist $(\beta=0,22)$ und diese wiederum positiv mit Lernzielorientierungen $(\beta=0,42)$ und negativ mit der Arbeitsvermeidung $(\beta=-0,30)$ zusammenhängt. Der Effekt auf Kompetenz (pädagogisches Wissen, $\beta=0,15$ ) fällt für die intrinsische Berufswahlmotivation, vermittelt über die Hoffnung auf Erfolg und die Lernzielorientierung, positiv aus (König und Rothland 2013, S. 58). Die Furcht vor Misserfolg kann von dem Ausmaß vorhergesagt werden $(\beta=0,37)$, in dem das Lehramtsstudium als Verlegenheitslösung betrachtet wird. Verlegenheitslösung bedeutet hier, dass das Lehramtsstudium aus Mangel an Alternativen (z. B. lag keine Zulassung für das Wunschstudium vor) aufgenommen wurde, oder auch aus Unschlüssigkeit oder Unsicherheit bei der Studien- bzw. Berufswahl. Die Furcht vor Misserfolg hängt außerdem positiv mit der Arbeitsvermeidung zusammen. Der Effekt auf pädagogisches Wissen fällt hier negativ aus $(\beta=-0,10$, König und Rothland 2013, S. 58).

Da die Zusammenhänge zwischen der Leistungsmotivation und den Zielorientierungen selten untersucht wurden, sei an dieser Stelle noch auf Ergebnisse aus Studien mit Schüler*innen hingewiesen, Für Schüler*innen hat sich bislang eher gezeigt, dass Lernzielorientierungen nicht in einem Zusammenhang mit der Angst vor Misserfolg stehen (Spinath et al. 2002). Je höher aber die Hoffnung auf Erfolg ist, desto stärker ausgeprägt sind Lernzielorientierung und intrinsische Motivation (Dickhäuser et al. 2016) und desto besser sind auch die Schulleistungen (Steinmayr 
und Spinath 2009; Dickhäuser et al. 2016). Umgekehrt beeinflusst das Misserfolgsmotiv über eine ausgeprägtere Vermeidungs-, aber auch Annäherungs-Leistungszielorientierung Leistung bzw. Noten negativ (Steinmayr und Spinath 2009; Dickhäuser et al. 2016). Diese Ergebnisse können Hinweise auf mögliche, erwartbare Zusammenhänge bei Lehramtsstudierenden liefern,

Insgesamt versprechen die hier dargestellten Ergebnisse zur Leistungsmotivation und den Zielorientierungen eine lohnende Untersuchung der Zusammenhänge zwischen den beiden Konstrukten. Deutlich wird, dass sich zwischen der Hoffnung auf Erfolg, den Lernzielorientierung und der intrinsischen Berufswahlmotivation positive Zusammenhänge zeigen. Entsprechend positive Zusammenhänge zeigen sich auch für das Misserfolgsmotiv zu vor allem den Vermeidungs-Leistungszielen und der Tendenz zur Arbeitsvermeidung, sodass auch Profilgruppen mit entsprechenden Ausprägungen - wie ein Profil mit hohen Erfolgserwartungen und hoher Lernzielorientierung, oder einem mit einer ausgeprägten Furcht vor Misserfolg und einer damit einhergehenden hohen Arbeitsvermeidung - zu erwarten sind.

\subsection{Profile nach Lern-/Leistungszielorientierungen und Allgemeiner Leistungsmotivation}

Da es sich bei Zielorientierungen nicht um ein eindimensionales Konstrukt handelt, sondern Personen nachweislich multiple Ziele gleichzeitig verfolgen können (vgl. Pintrich 2000; Barron und Harackiewicz 2001; Valle et al. 2003), kann davon ausgegangen werden, dass auch Lehramtsstudierende unterschiedliche Ausprägungen in ihren Zielorientierungen aufweisen. Es finden sich Studien, die Profilanalysen der Lern- und Leistungsmotivation von Schüler*innen (vgl. Meece und Holt 1993; Köller 1998; Schwinger und Wild 2006; Tuominen-Soini et al. 2012; Watermann 2013), Referendar*innen (Fasching et al. 2010) sowie (angehenden) Lehrkräften (Affolter 2016) vornehmen. Klassifikationen von Studierenden nach ihren Zielorientierungen sind eher rar (vgl. z. B. Pastor et al. 2007).

Bei Profil- oder Clusterbildungen gilt es zu beachten, dass Profile und Cluster je nach Stichprobe und eingesetzten Instrumenten unterschiedlich ausfallen können (Schwinger und Wild 2006). Auch das Analyseverfahren der Profil- oder Clusterbildung spielt eine Rolle. Die Clusteranalyse ist eine variablenzentrierte Art der Typologiebildung, während die Latent Class oder Latent Profile Analyse (LPA) ein personenzentriertes Verfahren darstellt (Rost 2006; Geiser 2011). So ist es nicht überraschend, dass Studien zu Lern- und Leistungszielorientierungen eine unterschiedliche Anzahl von Gruppen identifizieren, die sich zwischen drei und sechs Profilen bewegen. Wormington und Linnenbrink-Garcia (2017, S. 421) haben in einer Metaanalyse von insgesamt 22 Studien zehn Profiltypen identifiziert: ,,[... five characterized by high pursuit of one or more goals [...], four characterized by low pursuit of one or more goals [...], and one characterized by average pursuit of all goals. “ Zusammenfassend lässt sich festhalten, dass das Profil mit ausgeprägten Lernzielen auch die signifikant höchste Motivation, das höchste Wohlbefinden und das höchste Engagement aufwies. Ein „Low All Goals“-Profil zeigte ähnlich hohe Werte für Wohlbefinden, das Profil mit hoher Arbeitsvermeidung dagegen die 
niedrigsten. Bezüglich der Leistung ließen sich keine signifikanten Unterschiede zwischen den Profilen ausmachen.

In einer Untersuchung mit Referendar*innen konnten Fasching et al. (2010) drei Cluster nachweisen, von denen eines adaptive Zielorientierungen aufwies und sich positiv von einem Profil mit hohen Leistungszielen und einem mit hoher Arbeitsvermeidung unterschied, und zwar in Leistung, Belastungserleben und der Einstellung gegenüber Hilfe. Pastor et al. (2007) haben ähnlich der vorliegenden Studie in einer LPA mit College-Studierenden, der ein vierfaktorielles Modell der Zielorientierungen zugrunde lag, sechs Profile identifiziert. Die Studie liefert insofern ein interessantes Ergebnis, als sich die Mehrheit der Studierenden in Profilen befand, die insgesamt hohe Werte der Zielorientierungen aufwiesen. Alle Profilgruppen zeigten außerdem hohe Lernzielorientierungen, und nur eine zeigte hohe Vermeidungsziele (Pastor et al. 2007, S. 38).

Die Leistungsmotive Hoffnung auf Erfolg und Angst vor Misserfolg wirken als Dispositionen für die Entwicklung von Zielorientierungen ebenfalls auf Verhalten und Erleben in Lern- und Leistungssituationen (vgl. Abb. 1). Sie stehen wie dargestellt mit den Zielorientierungen in einem theoretischen und empirischen Zusammenhang und werden deshalb gemeinsam mit den Lern- und Leistungszielen in der vorliegenden Studie herangezogen, um Profile von Lehramtsstudierenden zu generieren (Elliot und Church 1997; Brunstein und Heckhausen 2018). Sowohl Lernund Leistungszielorientierungen als auch die Leistungsmotivation weisen außerdem einen ,typologischen Charakter“ auf (Köller 1998, S. 142). Bislang haben Profilanalysen entweder nur ein Konstrukt berücksichtigt (Pastor et al. 2007; Watermann 2013), beide Konzepte getrennt behandelt (Köller 1998), oder Zielorientierungen gemeinsam mit weiteren Motiven und Persönlichkeitsmerkmalen typologisiert (Affolter 2016). Abb. 1 verdeutlicht das Zusammenspiel beider Konstrukte sowie die angenommenen Korrelationen.

\subsection{Zusammenhänge mit verwandten Konstrukten}

Bei den Lern- und Leistungsmotiven handelt es sich um proximale Faktoren, die die Studienleistung und somit Kompetenzentwicklung unmittelbar im Studium beeinflussen (König und Rothland 2013; Schiefele und Urhahne 2000). Die Leistungs- und Misserfolgsmotive sind ihnen vorgeordnet. Eingebettet in einen breiteren motivationalen Kontext hängen mit der Lern- und Leistungsmotivation im Studium zudem intrinsische und extrinsische Motive zusammen. Dazu gehören sowohl Studien- als auch Berufswahlmotive, die ,für die Leistungen in der ersten und zweiten Phase der Lehrerbildung“ bzw. den späteren Beruf motivieren (König und Rothland 2013, S. 45). Intrinsische Studien- und Berufswahlmotive sollten deshalb auch zu einer höheren intrinsischen Motivation im Studium, etwa bei der Beschäftigung mit den Studieninhalten führen (Künsting und Lipowsky 2011, S. 106). In Untersuchungen mit Lehramtsstudierenden gehen sie nachweislich mit einer höheren Studien- und Berufswahlzufriedenheit und mit unterstützenden Lernstrategien beim Wissenserwerb einher (Künsting und Lipowsky 2011; König und Rothland 2013). Intrinsische Motive hängen außerdem positiv mit dem Erfolgsmotiv und der Lernzielorientierung zusammen, die Verlegenheitslösung korreliert mit dem Misserfolgsmotiv und der Ar- 
beitsvermeidung (Elliot und Church 1997; Malmberg 2008; Künsting und Lipowsky 2011; König und Rothland 2013). Eine vorwiegend extrinsische Berufswahlmotivation suggeriert dagegen eher wenig Interesse für die Studieninhalte, sodass auch die Lern- und Leistungsmotivation, sich mit diesen Inhalten zu beschäftigen, geringer ausfallen dürfte (Künsting und Lipowsky 2011; vgl. auch die Befunde von Beckmann 2016). Aufgrund dieser Befundlage sollen anhand von intrinsischen, extrinsischen und sozial-pädagogischen Berufswahlmotiven des FIT-Choice-Modells (Richardson und Watt 2010) sowie der Zufriedenheit mit der Berufswahl die Profile anschließend validiert werden.

Neben den motivationalen Merkmalen soll in die Validierungsanalysen zusätzlich eine kognitive Komponente aufgenommen werden. Mit dem pädagogischen Wissen wird ein Kriterium berücksichtigt, das Kompetenz widerspiegelt und unmittelbarer durch Lern- und Leistungsverhalten und somit den Zielorientierungen (und der vorangestellten Leistungsmotive) prognostiziert werden kann (König und Rothland 2013).

\subsection{Die Rolle von Fächerdomänen}

Zielorientierungen können als dispositionale und somit relativ stabile oder als habituelle, also stärker situationsspezifische Merkmale konzeptualisiert werden (Pekrun 1988). Es stellt sich somit die Frage, ob sie kontextunabhängig oder domänenspezifisch betrachtet werden müssen (Köller 1998; Watermann 2013). Lern- und Leistungszielorientierungen von Lehramtsstudierenden zunächst als fächerübergreifend und somit dispositional zu verstehen liegt nahe, da das übergeordnete Berufsziel Lehrer*in dominiert (Watt et al. 2017; Glutsch et al. 2018), und zwar unabhängig von den studierten Fächern. Gleichzeitig kann vermutet werden, dass sich Lern- und Leistungszielorientierungen für verschiedene Fächer unterscheiden, da Zielorientierungen mit dem Selbstkonzept oder Interessen zusammenhängen und sich Personen sowohl in ihren Fähigkeiten als auch in ihrem Anstrengungsverhalten in verschiedenen Kontexten unterscheiden können (Sparfeldt et al. 2007, S. 166). Außerdem erfordern Fächerkulturen möglicherweise unterschiedliche Zielorientierungen (Schiefele 1978; Marsh et al. 1991).

Duda und Nicholls (1992, S. 291) verglichen bei Schüler*innen das Fach Sport mit allen anderen Fächern und zogen das Fazit, dass Ziele ein generalisiertes Konzept seien, das die Beschaffenheit persönlicher Erfolgskriterien reflektiert. Weitere Studien von Stipek und Gralinski (1996) der Fächer Mathematik und Sozialkunde, oder von Meece et al. (1988), die sechs naturwissenschaftliche Bereiche analysierten, kamen zu ähnlichen Ergebnissen. Auch Bong (2001, 2004) hat bei Schüler*innen der Sekundarstufen I und II Zusammenhänge zwischen Leistungszielorientierungen und Vermeidungs-Leistungszielorientierungen domänenübergreifend nachgewiesen. Für Lernzielorientierungen konnte die Autorin in der Sekundarstufe II allerdings Unterschiede zwischen den sprachlichen und mathematisch-naturwissenschaftlichen Fächern ausmachen.

Sparfeldt et al. (2007) untersuchten 1210 Schüler*innen (7.-10. Klasse) mit denselben vier Faktoren der Zielorientierungen wie in der vorliegenden Studie, aber fachspezifisch für verschiedene Schulfächer (u.a. Mathematik, Physik, Englisch) 


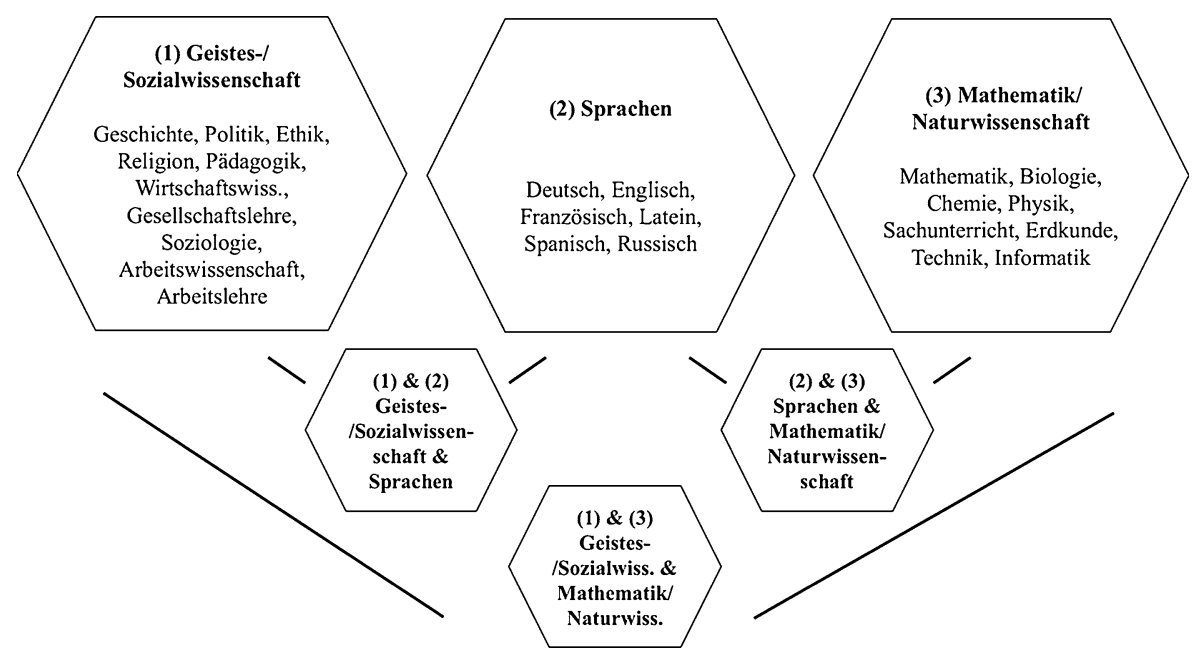

Abb. 2 Fächerklassifikation (Glutsch et al. 2018)

adaptiert. Für Lernziele konnte eine Fachspezifität belegt werden, tendenziell auch für Annäherungs-Leistungsziele. Für Vermeidungs-Leistungsziele und die Arbeitsvermeidung deuteten sich dagegen ,,eher globale bzw. fachübergreifende Strukturen an“ (Sparfeldt et al. 2007, S. 173). Dieses Ergebnis korrespondiert den Autor*innen zufolge mit der ,Nähe zwischen Lernzielen und intrinsischer Motivation bzw. Interesse [...].“ (Sparfeldt et al. 2007, S. 174). Zielorientierungen gelten einerseits also als „,bereichsunspezifische motivationale Merkmale“ (Köller 1998, S. 17), andererseits sprechen „Befunde der motivationspsychologischen Forschung [...] eher für die Domänenspezifität motivationaler Konstrukte“ (Watermann 2013, S. 29).

Mit Ausnahme der Studien von Sparfeldt et al. (2007), Bong (2001, 2004) sowie Stipek und Gralinski (1996) wurden in den bisherigen Untersuchungen nur wenige ausgewählte (häufig nur zwei) Fächerdomänen oder weit auseinanderliegende Fächer (wie Sport mit allen anderen Fächern) berücksichtigt. Alternativ wurden Fächerdomänen miteinander verglichen, die sich ähneln (naturwissenschaftliche Fächer). Hier könnte eine Ursache für Korrelationen zwischen den Zielorientierungen über verschiedene Fächer oder Fachbereiche hinweg liegen. Die Frage nach der Domänenspezifität motivationaler Merkmale bzw. der Zielorientierungen gilt somit nicht nur für Schüler*innen, sondern auch für Lehramtsstudierende noch als unbeantwortet. Dieses Forschungsdesiderat wird in der vorliegenden Studie aufgegriffen, indem sechs unterschiedliche Fächergruppen hinsichtlich ihrer Verteilung in den generierten Profilen untersucht werden (vgl. Abb. 2 sowie zu methodischen Details Abschn. 4.1). 


\section{Fragestellungen und Hypothesen}

Ziel dieser Studie ist es, Profile von Lehramtsstudierenden zu Beginn ihres Studiums basierend auf ihren Lern- und Leistungszielorientierungen sowie ihrer Leistungsmotivation zu identifizieren. Folgenden Forschungsfragen gehen wir dabei nach:

1. Welche Profile zeigen sich unter Einbeziehung der Lern- und Leistungszielorientierungen sowie Leistungs- und Misserfolgsmotive von Lehramtsstudierenden? Mittels einer LPA wird überprüft, ob sich Profile identifizieren lassen, die sich signifikant in der Lernzielorientierung, den Annäherungs- und Vermeidungs-Leistungszielorientierungen sowie in ihren Ausprägungen auf den Leistungsmotiven Hoffnung auf Erfolg und Furcht vor Misserfolg unterscheiden. Vor dem Hintergrund bisheriger Studien wie unter Punkt 2.4 dargestellt kann angenommen werden, dass sich mehrere Profile zeigen. Wir vermuten, dass sich ein Profil mit einer adaptiven bzw. günstigen Motivausprägung im Sinne einer hohen Hoffnung auf Erfolg und hoher Lernzielorientierung sowie niedrig ausgeprägten Vermeidungs-Leistungszielorientierungen und einer niedrigen arbeitsvermeidenden Haltung ergibt. Ein weiteres Profil mit mittleren Ausprägungen der Zielorientierungen und Leistungsmotivation sowie ein Profil mit eher maladaptiven bzw. ungünstigen Merkmalsausprägungen, das eine hohe Arbeitsvermeidung, ausgeprägte Vermeidungs-Leistungszielorientierungen und eine größere Furcht vor Misserfolg aufweist, sind ebenfalls zu erwarten (vgl. Pastor et al. 2007; Fasching et al. 2010; Wormington und Linnenbrink-Garcia 2017).

2. Lassen sich die Profile durch weitere motivationale sowie kognitive, berufsbezogene Merkmale validieren? Im Kontext des Kompetenzerwerbs und damit verbundener motivationaler Merkmale sind Zielorientierungen sowie Leistungsmotive in konzeptioneller Hinsicht mit Berufswahlmotiven verwandt (vgl. Köller 1998; Spinath et al. 2002). Nachweislich hängen intrinsische Motive positiv mit der Erwartung zusammen, erfolgreich zu sein (Erfolgsmotiv) sowie mit dem Streben nach eigener Kompetenzerweiterung (Lernzielorientierung). Wurde das Studium aus sogenannten Verlegenheitsgründen gewählt geht damit eine erhöhte Misserfolgserwartung und Tendenz zur Arbeitsvermeidung einher (Elliot und Church 1997; Malmber 2008; König und Rothland 2013; Beckmann 2016). Es wird daher angenommen, dass ein günstiges Profil mit hohen Lernzielorientierungen und hohem Erfolgsmotiv auch eine höhere intrinsische Berufswahlmotivation (intrinsischer Wert; Arbeit mit Kindern und Jugendlichen) sowie eine niedrige Ausprägung auf der Skala Verlegenheitslösung als ein mittleres oder weniger günstige Profile aufweist. Auch die wahrgenommene Lehrbefähigung, die von einem starken Erfolgsmotiv begünstigt wird, sollte bei einem adaptiven Profil höher ausgeprägt sein. Die sozial-gesellschaftliche Motivation, die altruistische Motive umfasst, steht vermutlich in keinem Zusammenhang mit den Lern- und Leistungszielen und könnte deshalb keine Unterschiede zwischen Profilen zeigen. Für die extrinsischen Motive (Berufliche Sicherheit; Vereinbarkeit von Familie und Beruf; Verlegenheitslösung) formulieren wir aufgrund bestehender Befunde die Annahme, dass sie in keinem Zusammenhang zu den günstigeren Profilen stehen und 
somit nur in Verbindung mit einem maladaptiven Profil bedeutsame Effekte aufweisen (Künsting und Lipowsky 2011).

Eine Profilanalyse mit Referendar*innen von Fasching et al. (2010) ergab, dass sich ein Profil mit adaptiven Lernzielorientierungen im Belastungserleben von weniger adaptiven Profilen positiv bedeutsam abhob und die Chance eines Ausbildungsabbruchs geringer war (vgl. auch Janke et al. 2015). Wir nehmen deshalb an, dass die Zufriedenheit mit der Berufswahl für ein Profil mit günstigen Zielorientierungen im Gegensatz zu anderen Profilen ebenfalls positiver ausfällt.

Schließlich wird das pädagogische Wissen als kognitives Merkmal von Lehramtsstudierenden untersucht, das nachweislich positiv mit der Lernzielorientierung und negativ mit der Arbeitsvermeidung korreliert (König und Rothland 2013). Auch hier wird erwartet, dass sich ein günstiges Motivprofil mit hohen Lernzeilen, aber auch (normativen) Annäherungs-Leistungszielen systematisch im pädagogischen Wissen von einem weniger adaptiven, arbeitsvermeidenden Profil unterscheidet.

3. Unterscheiden sich die Motivprofile in ihrer Zusammensetzung systematisch in Bezug auf die Fächerkombination der Studierenden? Obwohl bisherige Studienergebnisse bei Schüler*innen darauf hindeuten, dass zumindest die Lernzielorientierung fachspezifisch differenziert werden kann, treffen wir keine gerichtete Annahme für die Verteilung der Fächerkombinationen in den Profilgruppen. Die Studierenden befinden sich am Anfang ihres Studiums und die meisten sind vermutlich stark lernzielorientiert ausgerichtet (Pastor et al. 2007). Auch werden die Zielorientierungen und die Leistungsmotivation fächerübergreifend erfasst, sodass wir ein exploratives Vorgehen wählen, um mit den Ergebnissen das Forschungsfeld in einem ersten Schritt erweitern zu können (vgl. 2.3).

\section{Methode}

\subsection{Stichprobe}

Grundlage für die Studie ist eine Teilstichprobe des Projekts Entwicklung von berufsspezifischer Motivation und pädagogischem Wissen in der Lehrerausbildung $(E M W)^{1}$, das längsschnittlich neben pädagogischem Wissen die berufsspezifischen motivationalen Merkmale von Lehramtsstudierenden im ersten Semester und zu weiteren Zeitpunkten des Bachelor- und Master-Studiums und Berufseinstiegs erfasst. Zum ersten Messzeitpunkt wurden 18 Universitäten aus neun Bundesländern in Deutschland miteinbezogen (König et al. 2013). 4402 Lehramtsstudierende haben an der Studie im Rahmen einer Paper-Pencil-Befragung in Lehrveranstaltungen vor Ort teilgenommen. Vertreten sind fünf der sechs von der KMK definierten Lehramtstypen, Studierende des Lehramts für die beruflichen Schulen konnten nicht erreicht werden. Rücklaufquoten ließen sich nicht berechnen, da über das gewählte Erhebungsverfahren keine exakten Listen der Studierenden in den Veranstaltungen vorlagen (König et al. 2013, S. 563).

\footnotetext{
${ }^{1}$ Förderung durch die Rhein-Energie-Stiftung, Projektnummer: W-13-2-003 und W-15-2-003.
} 
Für die Analysen wurde eine Teilstichprobe von 1365 Studienanfänger*innen im ersten Fachsemester in den beiden fachlich spezialisierten Studiengängen für die Sekundarstufe I (KMK-Lehramtstyp 3) und Sekundarstufe I und II/Gymnasium (KMKLehramtstyp 4) in Deutschland verwendet. Der Studiengang für die Sekundarstufe I umfasst 40,7\% $(n=556)$ Studierende, jener für die Sekundarstufe I und II 59,3\% $(n=809) .70 \%$ der Studierenden sind weiblich, das durchschnittliche Alter beträgt $M=20$ Jahre $(S D=2,23)$. Die durchschnittliche Abiturnote der Sekundarstufen I und II-Studierenden ist mit $M=2,15(S D=0,59)$ besser als die der Sekundarstufen I-Studierenden mit $M=2,69(S D=0,48)$.

In beiden Lehramtstypen lassen sich die Studierenden aufgrund ihrer Fächerkombinationen aus zwei Fächern insgesamt sechs Gruppen zuordnen (vgl. Abb. 2). Die Einteilung erfolgt in (1) die Fächergruppe der Geistes-/Sozialwissenschaften (Geschichte, Politik, Ethik, Religion, Pädagogik, Wirtschaftswissenschaften, Gesellschaftslehre, Soziologie, Arbeitswissenschaft, Arbeitslehre; $n=64,4,7 \%$ ), (2) die Gruppe der Sprachwissenschaften (Deutsch, Englisch, Französisch, Latein, Spanisch, Russisch; $n=198,14,5 \%$ ), (3) die Gruppe der Mathematik/ Naturwissenschaften (Mathematik, Biologie, Chemie, Physik, Sachunterricht, Erdkunde, Technik, Informatik; $n=150,11 \%$ ) und in die daraus gebildeten drei weiteren gemischten Gruppen, die domänenübergreifend jeweils zwei Fächer aus den Gruppen (1) und (2) mit $n=480$ (35,2\%), den Gruppen (1) und (3) mit $n=230(16,9 \%)$ oder den Gruppen (2) und (3) mit $n=243$ (17,8\%) enthalten (vgl. Glutsch et al. 2018). In der vorliegenden Studie wurden bei der Gruppenbildung diejenigen Fächer berücksichtigt, die von den Studierenden zum ersten Messzeitpunkt mit mindestens einer Nennung angegeben wurden. Nicht integriert wurden Kunst, Musik und Sport, die sich inhaltlich keiner der übergeordneten Gruppen zuordnen lassen. Abb. 2 enthält die Fächergruppenzuordnung der sechs Fächergruppen mit jeweils zwei studierten Fächern.

\subsection{Instrumente}

\subsubsection{Lern-/Leistungszielorientierungen und Allgemeine Leistungsmotivation}

Die Lern- und Leistungszielorientierungen werden mit den Skalen zur Erfassung der Lern- und Leistungsmotivation für Studierende (SELLMO-ST) erfragt. Mit 31 Items (5-stufiges Antwortformat; 1 = stimmt gar nicht bis 5= stimmt genau) werden die vier Skalen Lernziele, Annäherungs-Leistungsziele, Vermeidungs-Leistungsziele und Arbeitsvermeidung erfasst (Spinath et al. 2002). Der Itemstamm für die Zielorientierungen lautet „Im Studium geht es mir darum, ....“. In die vorliegenden Analysen wurden nur 22 Items einbezogen, da die konfirmatorische Faktorenanalyse eine geringe Ladung einzelner Items der Skalen Lernzielorientierung und Annäherungs-Leistungsziele identifizierte $(<0,6)$ und eine geringe Itemtrennschärfe aufzeigte (Beispielitem für Lernzielorientierung: „Im Studium geht es mir darum, zum Nachdenken angeregt zu werden“). Die Autor*innen berichten bei der Testkonstruktion des SELLMO-ST über gute Reliabilitätswerte der Skalen, aber auch über Mehrfachladungen einiger Items bei der Faktorenanalyse (Spinath et al. 2002). Ein Überblick über die Items findet sich in Tab. 7 im Anhang. 
Die Allgemeine Leistungsmotivation (ALM) wird mit der Achievement Motives Scale (AMS; Gjesme und Nygard 1970²; dt. Übersetzung: Göttert und Kuhl 1980³) erfragt, die mit 17 Items (4-stufiges Antwortformat; $1=$ trifft gar nicht zu bis $4=$ trifft voll zu) die beiden Skalen Hoffnung auf Erfolg (8 Items; Beispielitem: „Es macht mir Spaß, an Problemen zu arbeiten, die für mich ein bisschen schwierig sind.“) und Furcht vor Misserfolg (9 Items; Beispielitem: „In etwas schwierigen Situationen, in denen viel von mir selbst abhängt, habe ich Angst zu versagen.") umfasst. Der Einleitungssatz für die Items der Allgemeinen Leistungsmotivation lautet „Im Folgenden möchten wir gerne erfahren, wie Sie mit herausfordernden Situationen allgemein umgehen. Wie ist es bei Ihnen?“. Hier wurden vier Items der Skala Hoffnung auf Erfolg aufgrund zu geringer Ladung nach faktorenanalytischer Prüfung ausgeschlossen. Die Ergebnisse sind ebenfalls in Tab. 7 im Anhang zu finden. Die deskriptiven Statistiken der SELLMO-ST- und ALM-Skalen sind in Tab. 2 dargestellt.

\subsubsection{Berufswahlmotivation und Zufriedenheit mit der Berufswahl}

Die Berufswahlmotivation und Zufriedenheit mit der Berufswahl der Studierenden wurden mithilfe der FIT-Choice-Skalen (Watt und Richardson 2007) in der deutschen Fassung (König und Rothland 2012; Watt et al. 2012) erhoben. Dabei messen ursprünglich elf Skalen (34 Items) die berufsspezifischen Motive (7-stufiges Antwortformat; $1=$ überhaupt nicht wichtig bis $7=$ äußerst wichtig). Die Skalen zur eigenen Lehr-Lernerfahrungen und dem positiven Einfluss Dritter ,stellen keine Motive im engeren Sinne dar" (König et al. 2018, S. 161) und werden deshalb ausgeklammert. Die drei sozial-altruistischen Faktoren ,Zukunft der Kinder und Jugendlichen mitgestalten“, „Soziale Benachteiligung aufheben“ und „Einen sozialen Beitrag für die Gesellschaft leisten“ wurden zu der Skala ,sozial-gesellschaftliche Motivation“ zusammengefasst (Watt et al. 2012; König et al. 2016). Somit werden zwei intrinsische (Intrinsischer Wert, Arbeit mit Kindern und Jugendlichen) und drei extrinsische Motivationsskalen (Verlegenheitslösung, Vereinbarkeit von Familie und Beruf, Berufliche Sicherheit), die wahrgenommene Lehrbefähigung sowie die altruistische bzw. sozial-gesellschaftliche Motivation in den Analysen berücksichtigt.

Die Zufriedenheit mit der Berufswahl (2 Items) gehört als eine von sechs Skalen zu den berufsbezogenen Überzeugungen im FIT-Choice-Modell (Watt und Richardson 2007). Ihr ist die Aufforderung vorangestellt „Zu Ihrer Entscheidung Lehrer/Lehrerin zu werden: Bitte beurteilen Sie, wie sehr die folgenden Aussagen auf Sie persönlich zutreffen!“ (7-stufiges Antwortformat; $1=$ überhaupt nicht bis $7=$ äußerst).

\footnotetext{
2 Gjesme, T., \& Nygard, R. (1970). Achievement-related motives: Theoretical considerations and construction of a measuring instrument (unveröffentlichter Bericht). Oslo: University of Oslo.

3 Göttert, R., \& Kuhl, J. (1980). LM-Fragebogen. Deutsche Übersetzung der AMS-Skala von Gjesme und Nygard (unveröffentlichtes Manuskript). Bochum: Ruhr-Universität Bochum.
} 


\subsubsection{Pädagogisches Wissen}

Das pädagogische Wissen wird in der vorliegenden Studie als kognitive Komponente professioneller Kompetenz betrachtet. Zur Erfassung wurde eine Kurzfassung des im Kontext der internationalen Vergleichsstudie entwickelten TEDS-M Testinstruments verwendet. Dieses umfasst 42 Items aus 13 Aufgaben mit den inhaltlichen Dimensionen ,Strukturierung von Unterricht“, ,Umgang mit Heterogenität“, ,,Klassenführung“ und „Motivierung und Leistungsbeurteilung“. In den Validierungsanalysen wird das pädagogische Wissen in Form einer Skala auf Grundlage des RaschModells eingesetzt (EAP-/WLE-Reliabilität: 0,76/0,74; Theta-Varianz: 0,67; weighted MNSQ: 0,88-1,09; vgl. dazu König und Rothland 2013), die bereits in vorangehenden Studien gebildet und hier genutzt wird. Die Lösungshäufigkeiten der im Wissenstest eingesetzten Aufgaben liegt bei Studienanfänger*innen im Durchschnitt bei etwa 30 bis $40 \%$ und steigt dann im Verlauf bis zum Ende der Ausbildung deutlich an (vgl. König 2012). Besonders schwierige Testaufgaben mussten aufgrund zu geringer Lösungshäufigkeiten von der Skalierung des Tests ausgeschlossen werden, „,von denen wir jedoch wissen, dass sie zur Messung des pädagogischen Wissens angehender Lehrer in einem fortgeschrittenen Stadium der Lehrerausbildung geeignet sind.“ (König und Rothland 2013, S. 61).

\subsection{Analysen}

Konfirmatorische Faktorenanalysen sowie Latent Profile Analysen (LPA) erfolgen im Strukturgleichungsansatz auf latenter Ebene mit Mplus 7 (Muthén und Muthén 2012). Die LPA ist ein personenzentriertes Verfahren der Datenanalyse (Rost 2006), das bei einer multivariaten Datengrundlage angewendet werden kann. Bei latenten Merkmalen wie dem motivationalen Konstrukt der Zielorientierungen erfolgt die Zuordnung von Personen mit bestimmten Antwortmustern zu den gefundenen Profilen mit einer bestimmten Wahrscheinlichkeit. Die Personen einer identifizierbaren Gruppe weisen alle dieselbe Wahrscheinlichkeit aller beobachteten Merkmale auf (Rost 2006, S. 277). Die Validierung der Profile anhand von Berufswahlmotiven, der Zufriedenheit mit der Berufswahl und dem pädagogischen Wissen erfolgt ebenfalls mit Mplus 7 in Form einer latenten Pfadanalyse. Fehlende Werte wurden mit dem FIML-Verfahren (Full Information Maximum Likelihood-Verfahren) in Mplus 7 geschätzt. Für die Verteilungsanalyse der Fächerkombinationen in den Profilen wurde ein Chi-Quadrat-Test mit SPSS durchgeführt.

\section{Ergebnisse}

\subsection{Konfirmatorische Faktorenanalysen und Korrelationen}

Die Ergebnisse der konfirmatorischen Faktorenanalyse für den SELLMO-ST zeigt einen ausreichenden Modell-Fit $\left(\chi^{2} / \mathrm{df}=5,18 ; p<0,001\right.$; CFI = 0,92; RMSEA = 0,06; SRMR =0,04). Die Reliabilitätswerte sind für alle vier Faktoren akzeptabel bis sehr gut. Cronbachs Alpha liegt für die Skala „Lernzielorientierung“ bei 0,70, für die 
Tab. 1 Latente Korrelationen der ALM- und SELLMO-ST-Skalen

\begin{tabular}{llllll}
\hline & $\begin{array}{l}\text { Hoffnung } \\
\text { auf Erfolg }\end{array}$ & $\begin{array}{l}\text { Furcht vor } \\
\text { Misserfolg }\end{array}$ & Lernziele & $\begin{array}{c}\text { Annäherungs- } \\
\text { Leistungsziele }\end{array}$ & $\begin{array}{l}\text { Vermeidungs- } \\
\text { Leistungsziele }\end{array}$ \\
\hline $\begin{array}{l}\text { Furcht vor } \\
\text { Misserfolg }\end{array}$ & $-0,45^{* * *}$ & - & - & - & - \\
Lernziele & $0,44 * * *$ & $-0,22 * * *$ & - & - & - \\
Annäherungs- & $-0,12 * *$ & $0,24 * * *$ & $-0,16^{* * * *}$ & - & - \\
$\begin{array}{l}\text { Leistungsziele } \\
\text { Vermeidungs- }\end{array}$ & $-0,27 * * *$ & $0,46^{* * *}$ & $-0,27 * * *$ & $0,82 * * *$ & - \\
$\begin{array}{l}\text { Leistungsziele } \\
\text { Arbeitsvermeidung }\end{array}$ & $-0,43 * * *$ & $0,31 * * *$ & $-0,45^{* * * *}$ & $0,46 * * *$ & $0,59 * * *$ \\
\hline
\end{tabular}

Anmerkung: $* * p<0,01 ; * * * p<0,001$. Modell-Fit: $\chi^{2} / \mathrm{df}=3,64, p<0,001 ; \mathrm{CFI}=0,91$; RMSEA=0,05; $\mathrm{SRMR}=0,05$

„Annäherungs-Leistungszielorientierung“ bei 0,74, für die „Vermeidungs-Leistungszielorientierung“ bei 0,89 und für die „Arbeitsvermeidung“ bei 0,90. Der ModellFit der konfirmatorischen Faktorenanalyse für die ALM ist ebenfalls ausreichend $\left(\chi^{2} / \mathrm{df}=5,70 ; p<0,001 ; \mathrm{CFI}=0,94 ; \mathrm{RMSEA}=0,06 ; \mathrm{SRMR}=0,04\right)$. Cronbachs Alpha liegt für die „Hoffnung auf Erfolg“ bei 0,74 und für die „Furcht vor Misserfolg“ bei 0,87 und somit in einem guten Bereich.

Die latenten Korrelationen wurden für die SELLMO-ST-Skalen und die ALM dann zusätzlich in einem gemeinsamen Modell berechnet (Modell-Fit: $\chi^{2} / \mathrm{df}=3,64$, $p<0,001$; CFI: 0,91; RMSEA: 0,05; SRMR: 0,05; vgl. Tab. 1). Alle Korrelationen sind signifikant, wobei die höchste Korrelation zwischen den Annäherungs-Leistungsziel- und den Vermeidungs-Leistungszielorientierungen besteht $(0,82)$. Zwar geht es bei der Annäherungs-Leistungszielorientierung darum, nach außen positiv $\mathrm{zu}$ wirken und bei der Vermeidungs-Leistungszielorientierung darum, im Kontext von Kompetenz nach außen etwas zu verbergen. Dennoch zielen beide Konstrukte im Kern auf die Außenwirkung bzw. Performanz ab und sind häufig stark korreliert, sodass die hohen Korrelationen erwartbar, aber die Konstrukte dennoch voneinander trennbar sind (vgl. Senko und Harackiewicz 2005; Pugh et al. 2010).

Der niedrigste Zusammenhang findet sich zwischen den Lernzielen und den Annäherungs-Leistungszielen $(-0,16)$ und der Hoffnung auf Erfolg, die negativ mit der Annäherungs-Leistungszielorientierung korreliert $(-0,12)$. Die Hoffnung auf Erfolg korreliert nur mit den Lernzielen positiv $(0,44)$, mit allen anderen Zielorientierungen und der Furcht vor Misserfolg negativ $(-0,45)$ (vgl. auch Brunstein und Heckhausen 2018, S. 177). Die Ergebnisse für die Korrelationen der Zielorientierungen stimmen mit denjenigen überein, die sich für alle Lehrämter der Gesamtstichprobe in einer früheren Studie zum Zusammenhang von pädagogischem Wissen und berufsspezifischer Motivation zeigten (König und Rothland 2013).

\subsection{Deskriptive Ergebnisse}

Die Mittelwerte und Standardfehler der ALM- und SELLMO-ST-Skalen sind in Tab. 2 dargestellt. Die Ergebnisse zeigen insgesamt eine sehr hoch ausgeprägte Lernzielorientierung und im Vergleich weitaus geringer ausgeprägte Werte der Annäherungs- und Vermeidungs-Leistungsziele sowie der Arbeitsvermeidung. Die Werte 
Tab. 2 Deskriptive Statistiken der SELLMO-ST- und ALM-Skalen

\begin{tabular}{llll}
\hline Skala & M & SE & SD \\
\hline 1. Hoffnung auf Erfolg & 2,81 & 0,02 & 0,53 \\
2. Furcht vor Misserfolg & 2,05 & 0,02 & 0,53 \\
3. Lernziele & 4,44 & 0,02 & 0,50 \\
4. Annäherungs-Leistungsziele & 2,53 & 0,03 & 0,88 \\
5. Vermeidungs-Leistungsziele & 2,31 & 0,02 & 0,80 \\
6. Arbeitsvermeidung & 2,09 & 0,02 & 0,76 \\
\hline
\end{tabular}

Anmerkung: Skala ALM $=1-4$ und Skala SELLMO-ST $=1-5$

stimmen mit den Ergebnissen vorangehender Studien zu den Lernzielorientierungen weitgehend überein (König und Herzmann 2011; König und Rothland 2013).

\subsection{Bildung von Profilen}

In der vorliegenden Studie wurden latente Modelle mit zwei bis sechs Klassen getestet, Varianzen wurden frei geschätzt. Als statistische Kriterien für die Modellauswahl wurden die informationstheoretischen Kriterien, die den relativen Modellfit beschreiben, AIC (Akaike Information Criterion, Akaike 1987), BIC (Bayesian Information Criterion, Schwarz 1978) und aBIC (adjusted Bayesian Information Criterion, Sclove 1987) verwendet, für die im Modellvergleich als Referenz der geringere Wert nach Abflachen genutzt wird. Für den Modellvergleich wurden auch die Likelihood-Quotienten-Tests aLMR (adjusted Lo-Mendell-Rubin Likelihood Ratio Test) und PBLRT (Parametric Bootstrap Likelihood Ratio Test) herangezogen (Rost 2006; Nylund et al. 2007). Den statistischen Ergebnissen zufolge lässt sich eine Dreiklassenlösung als bestes Modell identifizieren (vgl. Tab. 3). Die ModellFits geben nach den Kriterien keine eindeutigen Hinweise, dass mehr als drei Profile eine bessere Lösung darstellen würden. Zwar flacht der BIC erst nach der Vierklassenlösung ab, für die Dreiklassenlösung spricht aber vor allem, dass der aLMR bei der Vierklassenlösung als Test für den Modellvergleich gegenüber einem sparsameren Modell (k-1) nicht mehr signifikant wird (Nylund et al. 2007). Auch liegt die Klassenzugehörigkeitswahrscheinlichkeit bei der Dreiklassenlösung im Gegensatz zu einer Vierklassenlösung für alle drei Klassen bei mindestens 0,80. Um die Ergebnisse abzusichern, wurden noch ein fünftes und sechstes Modell berechnet. Sie liefern keine besseren Modellfits und bestätigen die Entscheidung für das Dreiklassenmodell (Loglikelihood = -6.088.100, $d f=33, \mathrm{aBIC}=12.279,33$, Entropy = 0,654), das daher für die weiteren Analysen herangezogen wird.

Die drei generierten Profile sind in Abb. 3 dargestellt. Das erste Profil 1 umfasst $32 \%(n=401)$ der Studierenden, das zweite Profil 50\% $(n=637)$ und das dritte Profil enthält $18 \%(n=227)$ der Studierenden. Die Profile unterschieden sich auf allen Skalen der Zielorientierungen und Leistungsmotivation signifikant (Tab. 4). Die aufgeklärte Varianz (partielles $\eta^{2}$ ) beträgt zwischen 10 und $72 \%$ und zeigt, dass tatsächlich diskriminierende Profile vorliegen. Die Lernzielorientierung und Hoffnung auf Erfolg, die für alle drei Profile etwa gleich stark ausgeprägt sind, weisen die geringste Varianz auf. Die größte Varianz zeigt sich in der Zielorientierung der Ver- 
Tab. 3 Informationskriterien für 2- bis 6-Klassenlösungen der Latenten Profilanalyse

\begin{tabular}{lllllll}
\hline $\begin{array}{l}\text { Anzahl Latenter } \\
\text { Profile }\end{array}$ & AIC & BIC & aBIC & aLMR & PBLRT & Entropy \\
\hline 2 & $12.429,18$ & $12.526,89$ & $12.466,54$ & $909,08^{* * *}$ & $-6659,22^{* * * *}$ & 0,668 \\
3 & $12.228,20$ & $12.361,91$ & $12.279,33$ & $210,76^{* *}$ & $-6195,59^{* * * *}$ & 0,654 \\
4 & $12.111,83$ & $12.281,54$ & $12.176,72$ & 127,82 & $-6088,10^{* * *}$ & 0,679 \\
5 & $12.052,33$ & $12.258,05$ & $12.130,99$ & 72,06 & $-6022,91^{* * *}$ & 0,665 \\
6 & $11.982,25$ & $12.223,96$ & $12.074,67$ & 82,44 & $-5986,17^{* * *}$ & 0,647 \\
\hline
\end{tabular}

$* * p<0,01, * * * p<0,001$

Tab. 4 Mittelwerte, Standardabweichungen der Profilgruppen und Ergebnisse der MANOVA

\begin{tabular}{llllll}
\hline $\begin{array}{l}\text { ALM- und SELLMO-ST- } \\
\text { Skalen }\end{array}$ & $M(S D)$ & & & $F(2,1024)$ & $\begin{array}{l}\text { Partielles } \\
\eta^{2}\end{array}$ \\
& Profil 1 & Profil 2 & Profil 3 & & \\
\hline 1. Hoffnung auf Erfolg & $2,97(0,53)$ & $2,83(0,47)$ & $2,49(0,47)$ & $55,76^{* * * *}$ & 0,10 \\
2. Furcht vor Misserfolg & $1,81(0,50)$ & $2,01(0,44)$ & $2,54(0,46)$ & $144,42^{* * *}$ & 0,22 \\
3. Lernziele & $4,61(0,41)$ & $4,42(0,47)$ & $4,13(0,58)$ & $62,09 * * *$ & 0,11 \\
$\begin{array}{l}\text { 4. Annäherungs-Leis- } \\
\text { tungsziele }\end{array}$ & $1,77(0,65)$ & $2,81(0,65)$ & $3,31(0,65)$ & $419,19 * * *$ & 0,45 \\
$\begin{array}{l}\text { 5. Vermeidungs-Leis- } \\
\text { tungsziele }\end{array}$ & $1,49(0,35)$ & $2,52(0,45)$ & $3,37(0,47)$ & $1303,84 * * *$ & 0,72 \\
6. Arbeitsvermeidung & $1,53(0,52)$ & $2,16(0,58)$ & $2,96(0,65)$ & $373,08^{* * * *}$ & 0,42 \\
\hline
\end{tabular}

Anmerkung: $* * * p<0,001$; Ergebnisse der multivariaten Varianzanalyse und Post-hoc-Tests mit Bonferroni-Korrektur

meidungs-Leistungsziele mit $72 \%$, gefolgt von Annäherungs-Leistungszielen (45\%) und Arbeitsvermeidung (42\%).

Die Studierenden der drei Profile können als (1.) Lernzielorientierte, (2.) Lernund Leistungszielorientierte und (3.) Indifferente bzw. Arbeitsvermeidende charakterisiert werden. Das erste Profil weist die höchste Hoffnung auf Erfolg sowie die niedrigste Furcht vor Misserfolg auf. Die Lernzielorientierung ist am stärksten ausgeprägt, die Annäherungs- und Vermeidungs-Leistungszielorientierungen sind wie die Arbeitsvermeidung sehr niedrig. Hier handelt es sich um ein stark lernzielorientiertes Profil, dem die Demonstration der eigenen Leistung nicht wichtig zu sein scheint. Es ist mit $32 \%$ am zweithäufigsten vertreten und zeigt adaptive Zielorientierungen und positive Leistungsmotive.

Das zweite Profil der Lernziel- und Leistungszielorientierten strebt verglichen mit dem ersten stärker nach Anerkennung von außen. Personen dieses Profils weisen ähnlich hohe Werte auf den Leistungsmotiven und Lernzielen auf wie diejenigen in Profil 1, haben aber eine höhere Annäherungs-Leistungsziel- sowie VermeidungsLeistungszielorientierung. Profil 2 ist außerdem stärker arbeitsvermeidend. Mit 50\% ist es das größte Profil unter den Studierenden.

Das dritte Profil der Indifferenten zeichnet sich zwar ebenfalls durch eine hohe Lernzielorientierung aus, ist aber stark außenorientiert und arbeitsvermeidend. Bei diesem Profil sind die Hoffnung auf Erfolg und die Furcht vor Misserfolg gleich stark ausgeprägt. Die Hoffnung auf Erfolg ist geringer, die Furcht vor Misserfolg 


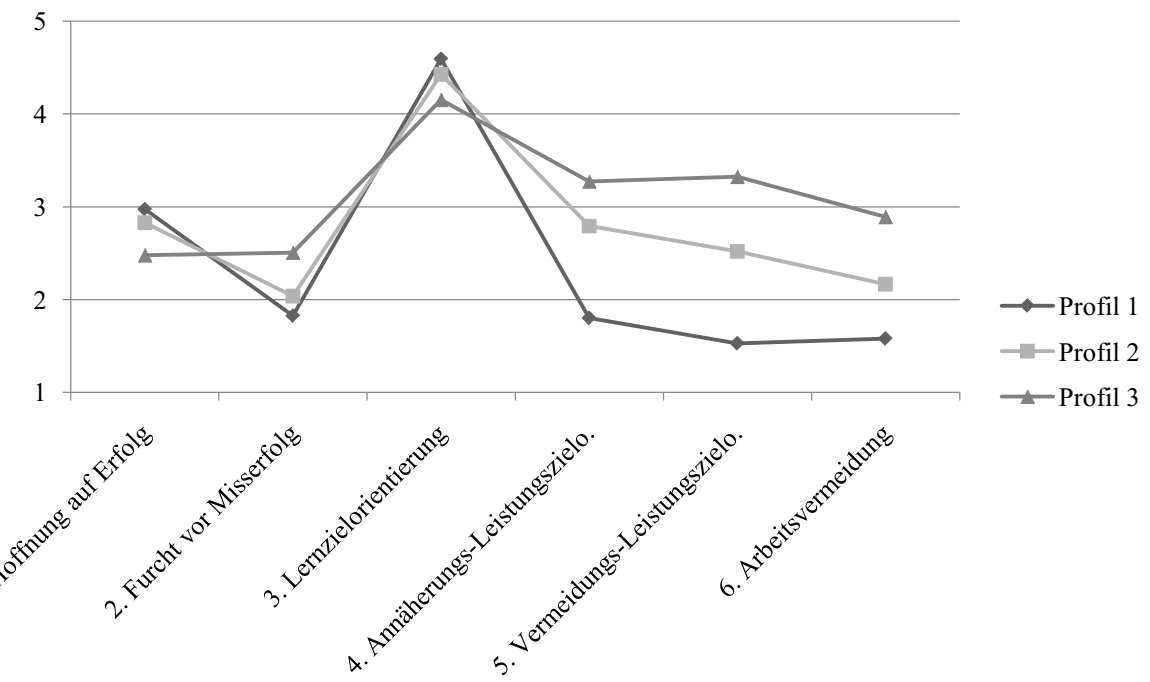

Abb. 3 Skalenmittelwerte für die drei Profile. Anmerkung: Skala ALM=1-4 und SELLMO-ST=1-5

dafür stärker ausgeprägt als in den anderen beiden Profilen. Bei Profil 3 ist die Vermeidungs-Leistungszielorientierung etwas stärker als die Annäherungs-Leistungszielorientierung. Die Arbeitsvermeidung ist bei diesem Profil im Vergleich zu den anderen beiden am höchsten. Mit $18 \%$ ist es das kleinste und in den Motivausprägungen ein eher ungünstiges Profil. Diese Profilgruppe enthält verstärkt Personen, die multiple Ziele gleichzeitig verfolgen.

\subsection{Fächerunterschiede in den Profilen}

Anhand eines Chi-Quadrat-Tests wurde für die zweite Fragestellung untersucht, ob die Fächerkombinationen bezüglich der Profile unabhängig verteilt sind (vgl. Tab. 5). Dabei kann für die sechs Fächergruppen keine signifikante Abhängigkeit zu einem der drei Profile festgestellt werden $\left(\chi^{2}(10)=9355, p=0,50\right)$. Die Fächerkombinationen machen für die Profilzusammensetzung keinen Unterschied. Somit können Zielorientierungen und Leistungsmotive in ihrer unterschiedlichen Ausprägung in den Profilen eher als fächerübergreifend betrachtet werden. ${ }^{4}$

\footnotetext{
4 Eine weitere Prüfung erfolgte mittels einer multinomialen logistischen Regression (Referenzkategorie $=$ Profil 3), in die zusätzlich zu den Fächerkombinationen (5 Dummy-Variablen; Referenzkategorie $=(1)$ Geistes-/Sozialwissenschaften) die Kontrollvariablen Lehramtsstudiengang (1 = Sekundarstufe II), Geschlecht $(1=$ weiblich $)$ und die Abiturnote als unabhängige Variablen einbezogen wurden. Auch hier zeigte sich kein signifikantes Ergebnis für das Gesamtmodell $\left(\chi^{2}(16)=22,77, p=0,12\right)$.
} 
Tab. 5 Verteilung der Fächergruppen in den Profilen

\begin{tabular}{|c|c|c|c|c|c|c|c|c|c|c|c|c|}
\hline \multirow{3}{*}{$\begin{array}{l}\text { Profile } \\
\text { Profil } 1\end{array}$} & \multicolumn{12}{|c|}{ Fächergruppen } \\
\hline & \multicolumn{2}{|c|}{$\begin{array}{l}\text { Geistesw./ } \\
\text { Sozialw }\end{array}$} & \multicolumn{2}{|c|}{ Sprachen } & \multicolumn{2}{|c|}{$\begin{array}{l}\text { Mathe/ } \\
\text { NaWi }\end{array}$} & \multicolumn{2}{|c|}{$\begin{array}{l}\text { Geistes./Soz } \\
\& \text { Sprachen }\end{array}$} & \multicolumn{2}{|c|}{$\begin{array}{l}\text { Geistes./Soz } \\
\& \text { Mathe/ } \\
\text { NaWi }\end{array}$} & \multicolumn{2}{|c|}{$\begin{array}{l}\text { Sprachen \& } \\
\text { Mathe/NaWi }\end{array}$} \\
\hline & 18 & $29,5 \%$ & 51 & $29 \%$ & 40 & $29,9 \%$ & 155 & $34,1 \%$ & 68 & $31,6 \%$ & 69 & $30,8 \%$ \\
\hline Profil 2 & 31 & $50,8 \%$ & 83 & $47,2 \%$ & 75 & $47,2 \%$ & 217 & $47,7 \%$ & 114 & $53 \%$ & 117 & $52,2 \%$ \\
\hline Profil 3 & 12 & $19,7 \%$ & 42 & $23,9 \%$ & 19 & $23,9 \%$ & 83 & $18,2 \%$ & 33 & $15,3 \%$ & 38 & $17 \%$ \\
\hline Gesamt & 61 & $100 \%$ & 176 & $100 \%$ & 134 & $100 \%$ & 455 & $100 \%$ & 215 & $100 \%$ & 224 & $100 \%$ \\
\hline
\end{tabular}

\subsection{Profilvalidierung durch Berufswahlmotive, berufsbezogene Überzeugung und pädagogisches Wissen}

Für die Profilvalidierung, die wir in der dritten Fragestellung thematisieren, wurde eine latente Pfadanalyse durchgeführt. Die extremeren bzw. äußeren Profilgruppen 1 und 3 wurden als unabhängige (Dummy-)Variablen mit Profil 2 als Referenzgruppe, und die sieben Berufswahlmotive, die Zufriedenheit mit der Berufswahl sowie das pädagogische Wissen als abhängige Variablen einbezogen. Geschlecht, Lehramt und die Abiturnote wurden als Kontrollvariablen verwendet. ${ }^{5}$ Der Modell-Fit ist ausreichend $\left(\chi^{2} / \mathrm{df}=4,8, p<0,001, \mathrm{CFI}=0,92, \mathrm{RMSEA}=0,06, \mathrm{SRMR}=0,04\right)$. Die Ergebnisse (Tab. 6) zeigen, dass die drei Profile systematisch fast alle Berufswahlmotivationsskalen sowie die Zufriedenheit mit der Berufswahl signifikant vorhersagen können. Erwartungsgemäß hängt das günstige Profil 1 (hohe Lernziele, niedrige Leistungsziele, geringe Arbeitsvermeidung) im Vergleich zu Profil 2 (höhere Leistungszielorientierungen, höhere Arbeitsvermeidung) bedeutsam mit einer größeren intrinsischen Motivation sowie niedrigeren Wahrscheinlichkeit zusammen, das Studium aus Verlegenheit gewählt zu haben. Auch fallen die wahrgenommene Lehrbefähigung und die Zufriedenheit mit der Berufswahl für das adaptive Profil 1 wie erwartet signifikant hoch aus. Zusätzlich zu unseren Annahmen zeigt sich, dass Profil 2 die extrinsische Motivation bedeutend höher vorhersagt.

Für die Beziehung zur sozial-gesellschaftlichen Motivation findet sich zwar zwischen Profil 1 und 2 kein signifikanter Unterschied, aber das ungünstige Profil 3 hat im Vergleich mit dem mittleren Profil 2 einen signifikant negativen Einfluss auf die sozial-gesellschaftliche Motivation. Auch im pädagogischen Wissen unterscheidet sich der Einfluss von Profil 1 nicht signifikant von dem des größten Profils 2, was darauf hindeutet, dass Leistungsziele im Rahmen von Kompetenz oder Leistung keine ungünstigere Rolle spielen als Lernziele, vor allem nicht in Verbindung mit hohen Lernzielen. Profil 3 steht mit einem signifikant geringeren pädagogischen Wissen als Profil 2 im Zusammenhang. Zudem fallen die Unterschiede zwischen

\footnotetext{
5 Es liegen keine exakten Listen der Veranstaltungsteilnehmer*innen vor bzw. konnten während des Erhebungsverfahrens nicht eingesehen oder aus organisatorischen Gründen den Teilnehmern nicht individuell zugeordnet werden, sodass eine genestete Datenstruktur nur für die verschiedenen Universitätsstandorte (18) kontrolliert werden kann. Diese genestete Datenstruktur auf Standortbasis haben wir im Strukturgleichungsmodell (Pfadanalyse) ergänzend getestet und können dieselben Ergebnisse berichten. Außer für den RMSEA, der sich um lediglich den Wert 0,01 verschlechtert, ändern sich weder die FIT-Werte noch die Regressionsgewichte.
} 
Tab. 6 Standardisierte Regressionskoeffizienten der latenten Pfadanalyse

\begin{tabular}{|c|c|c|c|c|c|c|c|c|}
\hline $\begin{array}{l}\text { Wahrge- } \\
\text { nommene } \\
\text { Lehrbe- } \\
\text { fähigung }\end{array}$ & $\begin{array}{l}\text { Intrin- } \\
\text { sischer } \\
\text { Wert }\end{array}$ & $\begin{array}{l}\text { Verle- } \\
\text { genheits- } \\
\text { lösung }\end{array}$ & $\begin{array}{l}\text { Beruf- } \\
\text { liche } \\
\text { Sicher- } \\
\text { heit }\end{array}$ & $\begin{array}{l}\text { Verein- } \\
\text { barkeit } \\
\text { Familie/ } \\
\text { Beruf }\end{array}$ & $\begin{array}{l}\text { Sozial- } \\
\text { gesell. } \\
\text { Moti- } \\
\text { vation }\end{array}$ & $\begin{array}{l}\text { Arbeit } \\
\text { Kinder \& } \\
\text { Jugend- } \\
\text { liche }\end{array}$ & $\begin{array}{l}\text { Pädago- } \\
\text { gisches } \\
\text { Wissen }\end{array}$ & $\begin{array}{l}\text { Zufrie- } \\
\text { denh. } \\
\text { Berufs- } \\
\text { wahl }\end{array}$ \\
\hline Profil $10,174 *$ & $0,151^{*}$ & $-0,295^{*}$ & $-0,295^{*}$ & $-0,339^{*}$ & 0,114 & $0,156^{*}$ & $-0,017$ & $0,148^{*}$ \\
\hline Profil $3-0,393^{*}$ & $-0,579 *$ & $0,780 *$ & $0,186^{*}$ & $0,243^{*}$ & $-0,373^{*}$ & $-0,387^{*}$ & $-0,208 *$ & $-0,574^{*}$ \\
\hline
\end{tabular}

Anmerkung: ${ }^{*} p<0,05$; Profil 1 und 3 jeweils dichotom kodiert, Referenzkategorie: Profil 2

Profil 2 und Profil 3 für den Effekt auf die Verlegenheitslösung, den intrinsischen Wert und die Zufriedenheit mit der Berufswahl am stärksten aus. Die Befunde unterstützen die Annahme eines positiven Zusammenhangs zwischen Lernzielen und Leistungsmotiv mit intrinsischer Motivation sowie zwischen hohen Leistungs- und Vermeidungszielen und höherem Misserfolgsmotiv mit extrinsischer Motivation.

\section{Diskussion}

\subsection{Einordnung der Befunde}

Lern- und Leistungszielorientierungen sowie Leistungsmotive haben ,typologischen Charakter“" (Köller 1998, S. 142). Sie können daher in unterschiedlichen Ausprägungen Einfluss auf die Entwicklung der professionellen Kompetenz von Studierenden nehmen. Dies bestätigt auch die vorliegende Studie. Mittels einer LPA wurde zunächst untersucht, welche Profile von Zielorientierungen und Leistungsmotiven bei Studienanfänger*innen identifiziert werden können. Es haben sich drei Profile gezeigt, wie sie sich häufig für motivationale Merkmale bilden (König et al. 2018). Ihnen liegen unterschiedlich ausgeprägte und somit multiple Zielorientierungen sowie Leistungsmotive zugrunde. Die vier Faktoren der Zielorientierungen und die beiden Leistungsmotive lassen sich somit differenzieren und setzen sich in unterschiedlichen Konstellationen zusammen, wie dies bereits Elliot und Church (1997) in einem hierarchischen Modell beschreiben (vgl. auch Elliot und McGregor 1999).

Dabei ließ sich in Übereinstimmung mit anderen Studien wie vermutet ein Profil mit einer adaptiven bzw. günstigen Motivausprägung identifizieren, das etwa jeder dritte Studierende aufweist (Wormington und Linnenbrink-Garcia 2017; König et al. 2018). Auch ein Profil mit ungünstigen Merkmalsausprägungen ließ sich belegen. Es zeichnet sich vor allem durch die höchste Leistungszielorientierung und Arbeitsvermeidung aus und bildet die kleinste Gruppe unter den Studierenden, zu der aber immerhin noch jeder sechste Lehramtsstudierende der Stichprobe zählt. Entgegen unserer Annahme hat sich kein Profil mit durchgängig mittleren Zielausprägungen ergeben, wie es Wormington und Linnenbrink-Garcia (2017) in einer Metanalyse identifizierten. Das größte Profil 2 kommt mit einer mittleren Ausprägung auf den Annäherungs-Leistungszielen einem solchen Profil noch am nächsten.

Insgesamt weisen die Profilgruppen sehr hohe Lernzielorientierungen auf. Dieses Ergebnis steht mit den Befunden von Pastor et al. (2007) in Einklang, die unter Studierenden verschiedener Studiengänge durchgängig hohe Lernziele vorfan- 
den. Begründen ließe sich dies mit der Annahme einer generell hohen Motivation der Studierenden im ersten Semester, wenn das Bestreben darin liegt, neue Inhalte kennenzulernen und Kompetenzen zu erweitern. Auch wird der erfolgreiche Studienabschluss als langfristiges Ziel angestrebt. Die ausgeprägte Lernzielorientierung in allen Profilen könnte auch ein Grund dafür sein, dass sich wider Erwarten kein Profil mit durchgängig mittleren Ausprägungen auf den Zielorientierungen und der Leistungsmotivation identifizieren ließ.

Die Ausprägung der Leistungs- und Misserfolgsmotive erweist sich für die Profile 1 und 2 als sehr ähnlich und bestätigt damit Zusammenhänge mit den entsprechenden Ausprägungen der Zielorientierungen, wie sie bereits Elliot und Church (1997) beschreiben: Eine starkes Erfolgsmotiv ist in den Profilen mit hohen Lernzielorientierungen und mittleren Leistungszielorientierungen vertreten. Das Misserfolgsmotiv ist geringer ausgeprägt. Für das ungünstige Profil 3 stellt sich die Ausprägung umgekehrt dar. Hier finden sich Studierende, die eine stärkere Misserfolgsmotivation als Erfolgsmotivation aufweisen und damit auch höhere Leistungsziele und eine hohe Tendenz zur Arbeitsvermeidung (vgl. auch König und Rothland 2013).

Die Fächerdomänenzugehörigkeit spielt - zumindest am Anfang des Studiums keine Rolle für die Zusammensetzung der Profilgruppen. Es kann daher zunächst davon ausgegangen werden, dass Zielorientierungen und Leistungsmotivation, wie sie in der vorliegenden Studie erfasst wurden, fächerübergreifende Konzepte und eher dispositionale Merkmale darstellen. Dieses Ergebnis steht in Einklang mit den mehrheitlich domänenunspezifischen Befunden wie sie bei Meece et al. (1988), Stipek und Gralinski (1996), Köller (1998) oder Bong (2001) vorliegen.

Die identifizierten Profile konnten durch weitere motivationale Merkmale wie die berufsbezogene Motivation validiert werden. Wie erwartet hat das adaptive Profil 1 mit hohen Lernziel- und niedrigen Leistungszielorientierungen auch höhere intrinsische und niedrigere extrinsische Berufswahlmotive (Elliot und Church 1997; Malmberg 2008; Beckmann 2016). Für das pädagogische Wissen zeigt sich, dass das ungünstige Profil 3 eine schlechtere Leistung als das eher indifferente Profil 2 aufweist. Allerdings besteht zwischen Profil 2 und dem günstigen Profil 1 kein Unterschied. Dies deckt sich mit den Ergebnissen von Wormington und LinnenbrinkGarcia (2017), die keine Leistungsdifferenzen zwischen den Profilen, die allein Lernziele verfolgten, und solchen mit multiplen Lern- und Leistungszielorientierungen ausmachen konnten.

Ein wichtiges Ergebnis der Analysen manifestiert sich in der Zufriedenheit mit der Berufswahl. Die Profile unterscheiden sich bereits zu Beginn ihres Studiums deutlich. Die günstigeren Lern- und Leistungszielorientierungen und eine geringe arbeitsvermeidende Haltung der Personen in den Profilen 1 und 2 gehen erwartungsgemäß mit der größeren Zufriedenheit mit der Entscheidung für den Lehrberuf einher (vgl. auch Janke et al. 2015).

\subsection{Limitationen}

Limitationen der vorliegenden Studie liegen zum einen darin, dass Studierende der Lehrämter Sonderpädagogik sowie Grundschule (d.h. KMK-Typen 6 und 1) aufgrund der Analyse nach Fächerwahl, Studierende für das Lehramt an Berufsschulen 
(KMK-Typ 5) aufgrund fehlender Daten nicht einbezogen wurden. Vermutlich würde sich bei einer Analyse mit allen KMK-Lehramtstypen ebenfalls eine hohe Lernzielausprägung in den Profilen aufgrund des frühen Zeitpunktes im Studium zeigen. Ob sich andere Profilgruppen ergeben würden, kann auf der Grundlage bisheriger Befunde nicht prognostiziert werden. Außerdem wurden in der vorliegenden Analyse die Fächer Sport, Musik und Kunst ausgeklammert, sodass auch Personen mit diesen Fächern in Kombination mit anderen Fächern nicht vertreten waren. Aufgrund der Stichprobenzusammensetzung wären diese Fächer in Kombination mit anderen Fächern dann als Gruppen nicht mehr groß genug, um jeweils auf Unterschiede geprüft werden zu können. Im Zusammenhang mit diesen Fächern könnte sich aber beispielsweise eine stärkere Leistungszielausprägung bemerkbar machen und ein Effekt auf die Profilzusammensetzung festgestellt werden, da insbesondere in diesen Fächern bereits im Studium der Wettbewerb und die Demonstration der eigenen Leistung eine wichtige Rolle spielen.

Zusätzlich ist einschränkend anzumerken, dass die Zielorientierungen und Leistungsmotivation nicht fachspezifisch, sondern fächerübergreifend erfragt wurden. Es bleibt offen, welche Fächer beim Beantworten der Items für die Studierenden eine Rolle gespielt haben mögen. Interessant wäre eine weitere Spezifizierung der hier allgemein erfassten Zielorientierungen und Leistungsmotive, so beispielsweise eine differenziertere Unterteilung der Lern- und Leistungssituationen in Studium und Praxisphasen oder in fachdidaktische, fachspezifische und pädagogische Inhalte (vgl. Nitsche et al. 2011).

Bei den vorliegenden Ergebnissen handelt es sich um Querschnittsbefunde. Möglicherweise zeigen sich zu einem späteren Zeitpunkt des Studiums Unterschiede in der Fächergruppenverteilung, wenn die Anforderungen und Spezifika der verschiedenen Fächer deutlicher hervorgetreten sind. Über eine längsschnittliche Untersuchung könnte außerdem nachverfolgt werden, wie sich die eher günstigen und ungünstigen Profilgruppen in ihren Merkmalen, ihrer Zufriedenheit mit der Berufswahl und ihren Kompetenzen entwickeln.

\subsection{Implikationen für die Lehrer*innenbildung}

Leistungsmotivation und Zielorientierungen sind im Kontext der Lehrer*innenbildung noch relativ wenig erforscht, obwohl es sich um Konstrukte handelt, die die Motivation, das Verhalten und Erleben in Lern- und Leistungskontexten beschreiben können. Sie spielen also im Rahmen der professionellen Kompetenzentwicklung eine Rolle. Hier leistet die vorliegende Studie einen wichtigen Beitrag, indem Lehramtsstudierende im ersten Semester nach Profilen ihrer Lern- und Leistungsmotive untersucht werden. Unterschiedliche Ausprägungen der Lern- und Leistungsziele sowie Erfolgs- und Misserfolgsmotive zu Studienbeginn konnten identifiziert werden. Für Lehrkräfte im Beruf legen bislang vorliegende Befunde nahe, dass Zielorientierungen mit dem Erwerb professioneller Kompetenz, der Unterrichtsqualität, dem Unterrichtshandeln und dem Belastungserleben zusammenhängen (vgl. Punkte 2.2 und 2.3; für einen Überblick vgl. auch Daumiller 2018).

Bedenkt man den Verlauf des Studiums und die (schulpraktischen) Lerngelegenheiten, denen die Studierenden begegnen, so ist bei den Profilen mit hoher 
Leistungsmotivation und Lern- aber auch Annäherungsleistungszielorientierung eine positive Entwicklung bezüglich des Wissenserwerbs im Studium zu erwarten. Einflüsse wie Schulpraktika stellen Situationen dar, in denen sich vor allem die Zielorientierungen ändern können und die Studierenden weiter positiv oder negativ beeinflussen. Fasching et al. (2010) konnten allerdings belegen, dass sich eine Profilgruppe mit starker Arbeitsvermeidung während des Referendariats nicht veränderte, und schlussfolgern, dass im Rahmen der Lehrerbildung adaptive Zielorientierungen bewahrt und maladaptive modifiziert werden sollten. Bezüglich der zeitlichen Stabilität und der Diskussion um den State- oder Trait-Charakter von Zielorientierungen besteht somit weiterer Forschungsbedarf.

Zielorientierungen von Lehrkräften können sich auf die ihrer Schüler*innen auswirken. Eine hohe Lernzielorientierung im Unterricht befördert ein größeres Interesse am Lehren und somit auch Lernziele von Schüler*innen (vgl. Butler und Shibaz 2008; Retelsdorf et al. 2010; Dresel et al. 2013). Zusätzlich sinkt das Burnout-Risiko. Diese Zusammenhänge wären auch für Lehramtsstudierende im Studienverlauf relevant und noch zu untersuchen. Vor allem, da das ungünstige Profil $18 \%$ der Studierenden umfasst. Als lernförderlich erweist sich für Lehrkräfte außerdem die Einstellung zum Aufsuchen von Hilfe oder Unterstützung durch Kolleg*innen, die von lernzielorientierten Lehrkräften als nützlich und kompetenzsteigernd eingeschätzt wird (Butler 2007; Dickhäuser et al. 2007). Wie sich dies für Lehramtsstudierende darstellt, bleibt in der vorliegenden Studie offen, es kann aber von ähnlichen Zusammenhängen ausgegangen werden.

Insgesamt konnte gezeigt werden, dass Lehramtsstudierende in einem Profil mit hohen Leistungszielorientierungen bei gleichzeitig hohen Lernzielen gegenüber dem Profil mit hohen Lern-, aber geringen Leistungszielen eine schlechtere Passung an positiv besetzte Berufswahlmotive (Intrinsische Motivation; Wunsch mit Kindern und Jugendlichen zu arbeiten) und die Berufswahlzufriedenheit aufweisen. Vor allem mit Blick auf die weitere Entwicklung im Beruf, in dem positive Zielorientierungen von Lehrkräften gefordert werden, kann der Hinweis daher unterstützt werden, im Lehramtsstudium vor allem Lernzielorientierungen zu bestärken. In Bezug auf das pädagogische Wissen wurde dagegen deutlich, dass sich auch Leistungszielorientierungen als positiv erweisen. Vor diesem Hintergrund gilt es, weitere Forschung vor allem zur Entwicklung der motivationalen und kognitiven Merkmale in Zusammenhang mit der Leistungsmotivation und Lern- und Leistungszielen anzustreben.

Funding Open Access funding provided by Projekt DEAL.

Open Access Dieser Artikel wird unter der Creative Commons Namensnennung 4.0 International Lizenz veröffentlicht, welche die Nutzung, Vervielfältigung, Bearbeitung, Verbreitung und Wiedergabe in jeglichem Medium und Format erlaubt, sofern Sie den/die ursprünglichen Autor(en) und die Quelle ordnungsgemäß nennen, einen Link zur Creative Commons Lizenz beifügen und angeben, ob Änderungen vorgenommen wurden.

Die in diesem Artikel enthaltenen Bilder und sonstiges Drittmaterial unterliegen ebenfalls der genannten Creative Commons Lizenz, sofern sich aus der Abbildungslegende nichts anderes ergibt. Sofern das betreffende Material nicht unter der genannten Creative Commons Lizenz steht und die betreffende Handlung nicht nach gesetzlichen Vorschriften erlaubt ist, ist für die oben aufgeführten Weiterverwendungen des Materials die Einwilligung des jeweiligen Rechteinhabers einzuholen. 
Weitere Details zur Lizenz entnehmen Sie bitte der Lizenzinformation auf http://creativecommons.org/ licenses/by/4.0/deed.de.

\section{Anhang}

Tab. 7 Ergebnisse der CFA, standardisierte Faktorladungen und SE

\begin{tabular}{|c|c|c|c|c|}
\hline Variable & Item & Faktorladung & SE & $\mathrm{R}^{2}$ \\
\hline \multirow[t]{4}{*}{ Hoffnung auf Erfolg } & Item1_1 & 0,706 & 0,021 & 0,499 \\
\hline & Item1_2 & 0,672 & 0,022 & 0,451 \\
\hline & Item1_3 & 0,617 & 0,024 & 0,380 \\
\hline & Item1_4 & 0,587 & 0,025 & 0,344 \\
\hline \multirow[t]{9}{*}{ Furcht vor Misserfolg } & Item2_1 & 0,634 & 0,020 & 0,401 \\
\hline & Item2_2 & 0,737 & 0,016 & 0,543 \\
\hline & Item2_3 & 0,614 & 0,021 & 0,377 \\
\hline & Item2_4 & 0,632 & 0,020 & 0,399 \\
\hline & Item2_5 & 0,592 & 0,022 & 0,351 \\
\hline & Item2_6 & 0,649 & 0,020 & 0,421 \\
\hline & Item2_7 & 0,706 & 0,018 & 0,498 \\
\hline & Item2_8 & 0,539 & 0,024 & 0,291 \\
\hline & Item2_9 & 0,663 & 0,019 & 0,440 \\
\hline \multirow[t]{3}{*}{ Lernzielorientierung } & Item3_1 & 0,608 & 0,028 & 0,370 \\
\hline & Item3_2 & 0,667 & 0,027 & 0,445 \\
\hline & Item3_3 & 0,717 & 0,027 & 0,514 \\
\hline \multirow{3}{*}{$\begin{array}{l}\text { Annäherungs- } \\
\text { Leistungsziel- } \\
\text { orientierung }\end{array}$} & Item4_1 & 0,606 & 0,024 & 0,367 \\
\hline & Item4_2 & 0,798 & 0,018 & 0,637 \\
\hline & Item4_3 & 0,682 & 0,022 & 0,465 \\
\hline \multirow{8}{*}{$\begin{array}{l}\text { Vermeidungs- } \\
\text { Leistungsziel- } \\
\text { orientierung }\end{array}$} & Item5_1 & 0,640 & 0,020 & 0,410 \\
\hline & Item5_2 & 0,697 & 0,018 & 0,486 \\
\hline & Item5_3 & 0,707 & 0,017 & 0,499 \\
\hline & Item5_4 & 0,773 & 0,015 & 0,598 \\
\hline & Item5_5 & 0,768 & 0,015 & 0,590 \\
\hline & Item5_6 & 0,670 & 0,019 & 0,449 \\
\hline & Item5_7 & 0,701 & 0,018 & 0,492 \\
\hline & Item5_8 & 0,695 & 0,018 & 0,483 \\
\hline \multirow[t]{8}{*}{ Arbeitsvermeidung } & Item6_1 & 0,651 & 0,020 & 0,424 \\
\hline & Item6_2 & 0,664 & 0,019 & 0,441 \\
\hline & Item6_3 & 0,717 & 0,017 & 0,514 \\
\hline & Item6_4 & 0,807 & 0,013 & 0,651 \\
\hline & Item6_5 & 0,751 & 0,016 & 0,564 \\
\hline & Item6_6 & 0,715 & 0,018 & 0,511 \\
\hline & Item6_7 & 0,756 & 0,016 & 0,572 \\
\hline & Item6_8 & 0,724 & 0,017 & 0,524 \\
\hline
\end{tabular}

Anmerkung: Modell-Fit (CFA): $\chi^{2} / \mathrm{df}=3,64 ; p<0,001 ; \mathrm{CFI}=0,91 ; \mathrm{RMSEA}=0,05 ; \mathrm{SRMR}=0,05$ 


\section{Literatur}

Affolter, B. (2016). Engagement und Beanspruchung von Lehrpersonen in der Phase des Berufseintritts. Eine Erweiterung des JD-R-Modells durch motivationale Orientierungen und Persönlichkeitsmerkmale. München: TUM School of Education. Dissertation.

Akaike, H. (1987). Factor analysis and AIC. Psychometrika, 52(3), 317-332.

Ames, C. (1984). Achievement attributions and self-instructions under competitive and individualistic goal structures. Journal of Educational Psychology, 76(3), 478-487.

Atkinson, J. W. (1964). An introduction to motivation. Oxford: Van Nostrand.

Barron, K. E., \& Harackiewicz, J. M. (2001). Achievement goals and optimal motivation: testing multiple goals models. Journal of Personality and Social Psychology, 80(5), 706-722.

Beckmann, V. (2016). Studien- und Berufswahlmotive am Anfang des Lehramtsstudiums. In A. Boeger (Hrsg.), Eignung für den Lehrerberuf. Auswahl und Förderung (S. 115-135). Wiesbaden: Springer VS.

Bong, M. (2001). Between- and within-domain relations of academic motivation among middle and high school students: self-efficacy, task-value, and achievement goals. Journal of Educational Psychology, 93(1), 23-34.

Bong, M. (2004). Academic motivation in self-efficacy, task value, achievement goal orientations, and attributional beliefs. The Journal of Educational Research, 97(6), 287-298.

Brunstein, J.C., \& Heckhausen, H. (2018). Leistungsmotivation. In J. Heckhausen \& H. Heckhausen (Hrsg.), Motivation und Handeln (5. Aufl., S. 163-221). Heidelberg: Springer.

Butler, R. (2007). Teachers' achievement goal orientation and association with teachers' help-seeking: examination of a novel approach to teacher motivation. Journal of Educational Psychology, 99(2), 241-252.

Butler, R., \& Shibaz, L. (2008). Achievement goals for teaching as predictors of students' perceptions of instructional practices and students' help seeking and cheating. Learning and Instruction, 18(5), 453-467.

Daumiller, M. (2018). Motivation von Wissenschaftlern in Lehre und Forschung. Struktur, Eigenschaften, Bedingungen und Auswirkungen selbstbezogener Ziele. Wiesbaden: Springer.

Dickhäuser, O., Butler, R., \& Tönjes, B. (2007). Das zeigt doch nur, dass ich's nicht kann. Zielorientierung und Einstellung gegenüber Hilfe bei Lehramtsanwärtern. Zeitschrift für Entwicklungspsychologie und pädagogische Psychologie, 39(3), 120-126.

Dickhäuser, O., Dinger, F.C., Janke, S., Spinath, B., \& Steinmayr, R. (2016). A prospective correlational analysis of achievement goals as mediating constructs linking distal motivational dispositions to intrinsic motivation and academic achievement. Learning and Individual Differences, 50, 30-41.

Dresel, M., Fasching, M. S., Steuer, G., Nitsche, S., \& Dickhäuser, O. (2013). Relations be-tween teachers' goal orientations, their instructional practices and student motivation. Psychology, 4, 572-584.

Duda, J.L., \& Nicholls, J.G. (1992). Dimensions of achievement motivation in schoolwork and sport. Journal of Educational Psychology, 84(3), 290-299.

Dweck, C.S. (1986). Motivational processes affecting learning. American Psychologist, 41(10), 1040-1048.

Elliot, A. J., \& Church, M. (1997). A hierarchical model of approach and avoidance achievement motivation. Journal of Personality and Social Psychology, 72(1), 218-232.

Elliot, A. J., \& Harackiewicz, J.M. (1996). Approach and avoidance achievement goals and intrinsic motivation: a mediational analysis. Journal of Personality and Social Psychology, 70(3), 968-980.

Elliot, A. J., \& McGregor, H. A. (1999). Test anxiety and the hierarchical model of approach and avoidance achievement motivation. Journal of Personality and Social Psychology, 76(4), 628-644.

Elliot, A.J., \& McGregor, H. A. (2001). A $2 \times 2$ achievement goal framework. Journal of Personality and Social Psychology, 80(3), 501-519.

Fasching, M.S., Dresel, M., Dickhäuser, O., \& Nitsche, S. (2010). Goal orientations of teacher trainees: longitudinal analysis of magnitude, change and relevance. Journal for Educational Research Online, $2,9-33$.

Geiser, C. (2011). Datenanalyse mit Mplus. Eine anwendungsorientierte Einführung (2. Aufl.). Wiesbaden: VS.

Glutsch, N., König, J., \& Rothland, M. (2018). Die Berufswahlmotivation von angehenden Lehrkräften bei Eintritt in ihre Ausbildung - Unterschiede nach Fächerwahl? Zeitschrift für Pädagogik, 64(4), $461-485$. 
Harackiewicz, J. M., Barron, K. E., Carter, S. M., Lehto, A. T., \& Elliot, A. J. (1997). Predictors and consequences of achievement goals in the college classroom: Maintaining interest and making the grade. Journal of Personality and Social Psychology, 73(6), 1284-1295.

Janke, S., Nitsche, S., \& Dickhäuser, O. (2015). The role of perceived need satisfaction at work for teachers' work-related learning goal orientation. Teaching and Teacher Education, 47, 184-194.

Köller, O. (1998). Zielorientierungen und schulisches Lernen. Berlin: Waxmann.

König, J. (2012). Die Entwicklung von pädagogischem Unterrichtswissen: Theoretischer Rahmen, Testinstrument, Skalierung und Ergebnisse. In J. König \& A. Seifert (Hrsg.), Lehramtsstudierende erwerben pädagogisches Professionswissen. Ergebnisse der Längsschnittstudie LEK zur Wirksamkeit der Erziehungswissenschaftlichen Lehrerausbildung (S. 143-182). Münster: Waxmann.

König, J., \& Herzmann, P. (2011). Lernvoraussetzungen angehender Lehrkräfte am Anfang ihrer Ausbildung. Erste Ergebnisse aus der wissenschaftlichen Begleitung des Kölner Modellkollegs Bildungswissenschaften. Lehrerbildung auf dem Prüfstand, 4, 186-210.

König, J., \& Rothland, M. (2012). Motivations for choosing teaching as a career: effects on general pedagogical knowledge during initial teacher education. Asia-Pacific Journal of Teacher Education, 40, 289-315.

König, J., \& Rothland, M. (2013). Pädagogisches Wissen und berufsspezifische Motivation am Anfang der Lehrerausbildung. Zum Verhältnis von kognitiven und nicht-kognitiven Eingangsmerkmalen von Lehramtsstudierenden. Zeitschrift für Pädagogik, 59(1), 43-65.

König, J., Rothland, M., Darge, K., Lünnemann, M., \& Tachtsoglou, S. (2013). Erfassung und Struktur berufswahlrelevanter Faktoren für die Lehrerausbildung und den Lehrerberuf in Deutschland, Österreich und der Schweiz. Zeitschrift für Erziehungswissenschaft, 16(3), 553-577.

König, J., Rothland, M., Tachtsoglou, S., \& Klemenz, S. (2016). Change of teaching motivations among pre-service teachers in Austria, Germany, and Switzerland: Do in-school opportunities to learn matter? International Journal of Higher Education, 5(3), 91-103.

König, J., Drahmann, M., \& Rothland, M. (2018). Motivprofile von Studierenden zu Beginn der Lehrerbildung. Anwendung und Validierung eines personenzentrierten Ansatzes in Deutschland und Österreich. Zeitschrift für Bildungsforschung, 8(2), 153-171.

Künsting, J., \& Lipowsky, F. (2011). Studienwahlmotivation und Persönlichkeitseigenschaften als Prädiktoren für Zufriedenheit und Strategienutzung im Lehramtsstudium. Zeitschrift für Pädagogische Psychologie, 25(2), 105-114.

Kunter, M. (2014). Forschung zur Lehrermotivation. In E. Terhart, M. Rothland \& H. Bennewitz (Hrsg.), Handbuch der Forschung zum Lehrerberuf (Bd. 2, S. 698-711). Münster: Waxmann.

Kunter, M., Kleickmann, T., Klusmann, U., \& Richter, D. (2011). Die Entwicklung professioneller Kompetenz von Lehrkräften. In M. Kunter, J. Baumert, W. Blum, U. Klusmann, S. Krauss \& M. Neubrand (Hrsg.), Professionelle Kompetenz von Lehrkräften: Ergebnisse des Forschungsprogramms COACTIV (S. 55-68). Münster: Waxmann.

Malmberg, L.-E. (2008). Student teachers' achievement goal orientations during teacher studies: antecedents, correlates and outcomes. Learning and Instruction, 18(5), 438-452.

Marsh, H. W., Walker, R., \& Debus, R. (1991). Subject-specific components of academic self-concept and self-efficacy. Contemporary Educational Psychology, 16(4), 331-345.

Meece, J. L., \& Holt, K. (1993). A pattern analysis of students' achievement goals. The Journal of Educational Psychology, 85(4), 582-590.

Meece, J.L., Blumenfeld, P.C., \& Hoyle, R.H. (1988). Students' goal orientations and cognitive engagement in classroom activities. Journal of Educational Psychology, 80(4), 514-523.

Muthén, L. K., \& Muthén, B. O. (2012). Mplus User's Guide (7. Aufl.). Los Angeles: Muthén \& Muthén.

Nicholls, J. G. (1984). Achievement motivation: conceptions of ability, subjective experience, task choice, and performance. Psychological Review, 91(3), 328-346.

Nitsche, S., Dickhäuser, O., Fasching, M. S., \& Dresel, M. (2011). Rethinking teachers' goal orientations: conceptual and methodological enhancements. Learning and Instruction, 21(4), 574-586.

Nylund, K. L., Asparouhov, T., \& Muthen, B. (2007). Deciding on the number of classes in latent class analysis and growth mixture modeling: a monte carlo simulation study. Structural Equation Modeling: A Multidisciplinary Journal, 14, 535-569.

Pastor, D. A., Barron, K. E., Miller, B. J., \& Davis, S. L. (2007). A latent profile analysis of college students' achievement goal orientation. Contemporary Educational Psychology, 32(1), 8-47.

Paulick, I., Retelsdorf, J., \& Möller, J. (2013). Motivation for choosing teacher education: relations with teachers' achievement goals and instructional practices. International Journal of Educational Research, $61,60-70$.

Pekrun, R. (1988). Emotion, Motivation und Persönlichkeit. München: Psychologie Verlags Union. 
Pintrich, P. R. (2000). Multiple goals, multiple pathways: the role of goal orientation in learning and achievement. Journal of Educational Psychology, 92(3), 544-555.

Pugh, K. J., Linnenbrink-Garcia, L., Koskey, K.L. K., Stewart, V.C., \& Manzey, C. (2010). Motivation, learning, and transformative experience: a study of deep engagement in science. Science Education, 94(1), 1-25.

Retelsdorf, J., Butler, R., Streblow, L., \& Schiefele, U. (2010). Teachers' goal orientations for teaching: associations with instructional practices, interest in teaching, and burnout. Learning and Instruction, 20(1), 30-46.

Richardson, P. W., \& Watt, H. M. G. (2010). Current and future directions in teacher motivation research. In T. C. Urdan \& S. A. Karabenick (Hrsg.), The Decade Ahead: Applications and Contexts of Motivation and Achievement (Advances in Motivation and Achievement, Volume 16 Part B (S. 139-173). Bingley: Emerald.

Robbins, S., Lauver, K., Le, H., Davis, D., Langley, R., \& Carlstrom, A. (2004). Do psychological and study skill factors predict college outcome? A meta-analysis. Psychological Bulletin, 130(2), 261-288.

Rost, J. (2006). Latent-Class-Analyse. In F. Petermann \& M. Eid (Hrsg.), Handbuch der Psychologischen Diagnostik (S. 275-287). Göttingen: Hogrefe.

Rothland, M., \& Terhart, E. (2011). Eignungsabklärung angehender Lehrerinnen und Lehrer. Einführung in den Thementeil. Zeitschrift für Pädagogik, 57(5), 635-638.

Schiefele, H. (1978). Lernmotivation und Motivlernen. Grundzüge einer erziehungswissenschaftlichen Motivationslehre (2. Aufl.). München: Ehrenwirth.

Schiefele, U., \& Urhahne, D. (2000). Motivationale und volitionale Bedingungen der Studienleistung. In U. Schiefele \& K. Wild (Hrsg.), Interesse und Lernmotivation. Untersuchungen zu Entwicklung, Förderung und Wirkung (S. 183-205). Münster: Waxmann.

Schöne, C., Dickhäuser, O., Spinath, B., \& Stiensmeier-Pelster (2004). Zielorientierung und Bezugsnormorientierung: zum Zusammenhang zweier Konzepte. Zeitschrift für Pädagogische Psychologie, 18(2), 93-99.

Schwarz, G. (1978). Estimating the dimension of a model. The Annals of Statistics, 6, 461-464.

Schwinger, M., \& Wild, E. (2006). Die Entwicklung von Zielorientierung im Fach Mathematik von der 3. bis 5. Jahrgangsstufe. Zeitschrift für Pädagogische Psychologie, 20(4), 269-278.

Sclove, S.L. (1987). Application of model-selection criteria to some problems in multivariate analysis. Psychometrika, 52, 333-343.

Seifert, T.L., \& O'Keefe, B. (2001). The relationship of work avoidance and learning goals to perceived competence, externality and meaning. British Journal of Educational Psychology, 71, 81-92.

Senko, C., \& Dawson, B. (2017). Performance-approach goal effects depend on how they are defined: Meta-analytic evidence from multiple educational outcomes. Journal of Educational Psychology, 109(4), 574-598.

Senko, C., \& Harackiewicz, J. M. (2005). Regulation of achievement goals: The role of competence feedback. Journal of Educational Psychology, 97, 320-336.

Sparfeldt, J.R., Buch, S.R., Wirthwein, L., \& Rost, D.H. (2007). Zielorientierungen: Zur Relevanz der Schulfächer. Zeitschrift für Entwicklungspsychologie und Pädagogische Psychologie, 39, 165-176.

Spinath, B., \& Schöne, C. (2003). Ziele als Bedingungen von Motivation am Beispiel der Skalen zur Erfassung der Lern- und Leistungsmotivation (SELLMO). In J. Stiensmeier-Pelster \& F. Rheinberg (Hrsg.), Diagnostik von Motivation und Selbstkonzept (S. 29-40). Göttingen: Hogrefe.

Spinath, B., Stiensmeier-Pelster, J., Schöne, C., \& Dickhäuser, O. (2002). Skalen zur Erfassung der Lernund Leistungsmotivation (SELLMO). Göttingen: Hogrefe.

Steinmayr, R., \& Spinath, B. (2009). The importance of motivation as a predictor of school achievement. Learning and Individual Differences, 19(1), 80-90.

Stipek, D., \& Gralinski, J.H. (1996). Children's beliefs about intelligence and school performance. Journal of Educational Psychology, 88(3), 397-407.

Tönjes, B., \& Dickhäuser, O. (2009). Längsschnittliche Effekte von Zielorientierungen auf Faktoren des beruflichen Belastungserlebens im Lehrberuf. Zeitschrift für Entwicklungspsychologie und Pädagogische Psychologie, 41(2), 79-86.

Tönjes, B., Dickhäuser, O., \& Kröner, S. (2008). Berufliche Zielorientierungen und wahrgenommener Leistungsmangel bei Lehrkräften. Zeitschrift für pädagogische Psychologie, 22, 151-160.

Tuominen-Soini, H., Salmela-Aro, K., \& Niemivirta, M. (2012). Achievement goals orientations and academic well-being across the transition to upper secondary education. Learning and Individual Differences, 22(3), 290-305. 
Valle, A., Cabanach, R. G., Núnez, J. C., González-Pienda, J., Rodríguez, S., \& Pineiro, I. (2003). Multiple goals, motivation and academic learning. British Journal of Educational Psychology, 73(1), 71-87.

Watermann, R. (2013). Zielorientierung und der Zusammenhang mit der Schulleistung: Ein personenzentrierter Ansatz. In K. Schwippert, M. Bonsen \& N. Berkemeyer (Hrsg.), Schul- und Bildungsforschung. Diskussionen, Befunde und Perspektiven (S. 13-31). Münster: Waxmann.

Watt, H. M.G., \& Richardson, P.W. (2007). Motivational factors influencing teaching as a career choice: development and validation of the FIT-choice scale. Journal of Experimental Education, 75(3), 167-202.

Watt, H.M.G., Richardson, P.W., Klusmann, U., Kunter, M., Beyer, B., Trautwein, U., \& Baumert, J. (2012). Motivations for Choosing Teaching as a Career: An international comparison using the FITChoice scale. Teaching and Teacher Education, 28(6), 791-805.

Watt, H.M.G., Richardson, P. W., \& Morris, Z. M. (2017). Divided by discipline? Contrasting motivations, perceptions, and background characteristics of beginning Australian English and mathematics teachers. In H.M.G. Watt, P.W. Richardson \& K. Smith (Hrsg.), Global perspectives on teacher motivation (S. 349-376). New York: Cambridge University Press.

Wormington, S. V., \& Linnenbrink-Garcia, L. (2017). A new look at multiple goal pursuit: the promise of a person-centered approach. Educational Psychology Review, 29(3), 407-445. 